\title{
On a phase-field model for electrowetting
}

\author{
C. ECK \\ Institut für Angewandte Analysis und Numerische Simulation, Universität Stuttgart, \\ Pfaffenwaldring 57, 70569 Stuttgart, Germany \\ E-mail: christoph.eck@mathematik.uni-stuttgart.de \\ M. FONTELOS \\ Departamento de Matemáticas y Física Fundamental, C.S.I.C., \\ c/ Serrano, n 123, 28006 Madrid, Spain \\ E-mail:marco.fontelos@imaff.cfmac.csic.es \\ G. GRÜN \\ Department für Mathematik, Universität Erlangen-Nürnberg, \\ Martensstr. 3, 91058 Erlangen, Germany \\ E-mail: gruen@am.uni-erlangen.de \\ F. KLINGBEIL \\ Institut für Angewandte Mathematik, Universität Erlangen-Nürnberg, \\ Martensstr. 3, 91058 Erlangen, Germany \\ E-mail: klingbeil@am.uni-erlangen.de \\ O. VANTZOS \\ Institut für Numerische Simulation, Universität Bonn, \\ Endenicher Allee 60, 53115 Bonn, Deutschland \\ E-mail: orestis.vantzos@ins.uni-bonn.de
}

[Received 3 December 2007 and in revised form 19 February 2009]

\begin{abstract}
The term electrowetting is commonly used for phenomena where shape and wetting behavior of liquid droplets are changed by the application of electric fields. We develop and analyze a model for electrowetting that combines the Navier-Stokes system for fluid flow, a phase-field model of Cahn-Hilliard type for the movement of the interface, a charge transport equation, and the potential equation of electrostatics. The model is derived with the help of a variational principle due to Onsager and conservation laws. A modification of the model with the Stokes system instead of the NavierStokes system is also presented. The existence of weak solutions is proved for several cases in two and three space dimensions, either with non-degenerate or with degenerate electric conductivity vanishing in the droplet exterior. Some numerical examples in two space dimensions illustrate the applicability of the model.
\end{abstract}

2000 Mathematics Subject Classification: 35D05, 35D10, 35R35, 76M30.

Keywords: Electrowetting; phase-field model; Navier-Stokes system; Cahn-Hilliard model; free boundary problem in PDE; existence of weak solutions. 


\section{Introduction}

The wetting behavior of electrically conductive liquids in an insulating ambient fluid can be modified by the application of electric fields. This phenomenon is called electrowetting. As an example, a simple electrowetting experiment is sketched in Figure [ When a voltage is applied between an electrically conductive droplet and a hydrophobic or partially wettable substrate, the apparent contact angle is reduced and the droplet starts to spread. This effect has been discovered by Lippmann more than a century ago [23]. Based on his experimental findings, he suggested the following relation for the dependence of the contact angle $\Theta(V)$ on the voltage $V$ :

$$
\cos \Theta(V)=\cos \Theta(0)+\frac{\varepsilon_{0} \varepsilon}{2 d \gamma} V^{2} .
$$

Here, $\Theta(0)$ is the contact angle given by Young-Laplace theory [38], $\varepsilon_{0}$ and $\varepsilon_{0} \varepsilon$ are the dielectric permittivity in the vacuum and the dielectric layer between the droplet and the electrode respectively, $\gamma$ is the surface tension coefficient between the droplet and the ambient fluid, and $d$ the thickness of the insulating layer. Obviously, this formula can hold only in a small voltage regime. In particular, it claims that for sufficiently high voltages the complete wetting regime should be reached. Experiments by Vallet et al. [36] and by Mugele and Herminghaus [27], however, suggest the occurrence of saturation effects at small angles. Moreover, the formation of tiny satellite droplets at the contact line is observed which are connected to the bulk by liquid bridges [27]. A careful analysis by Mugele and Bührle [26] (see also [7]) indicates that the contact angle change predicted by Lippmann [23] is merely a macroscopic effect and that microscopically the Young angle persists. This result is confirmed by physical experiment (cf. [26]). In particular, contact angle changes consistent with the Lippmann formula (1.1) are observed only on lengthscales above the thickness of the insulator layer. All these results pertain to the stationary case, but to the best of our knowledge, the dynamical setting has not been theoretically addressed yet.

Besides these fundamental questions, the phenomenon of electrowetting is of major interest for technological applications related to microfluidics, more precisely the possibility of manipulating motion and shape of small amounts of fluid. A particular advantage compared to other methods studied recently is the enhanced flexibility of electrowetting since the control of the motion and wetting properties of fluids by suitably applied electric fields can be realized without valves, pumps or even fixed channels.

Important examples of technological applications are pixelated optical filters [30], adaptive lenses [21], [5], and curtain coating [6]. Similarly, fast switching electrowetting displays take
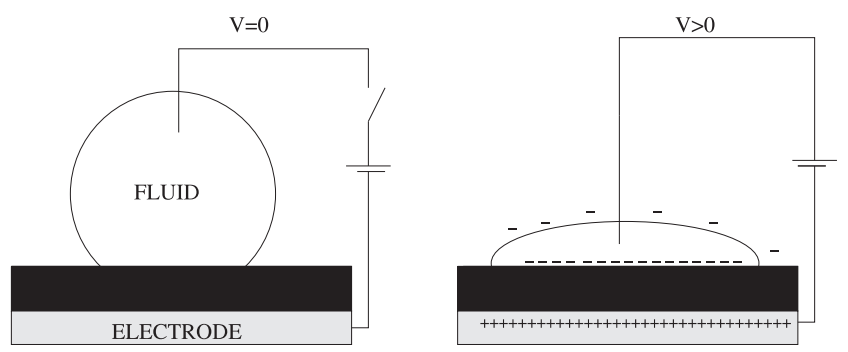

FIG. 1. Sketch of electrowetting phenomenon: A voltage difference is applied between the electrode in the water and a counter electrode present underneath the partially wettable insulator material. As a result of the voltage, the droplet spreads, i.e. the wettability of the surface increases strongly (see also [10] for a discussion of different experimental set-ups). 

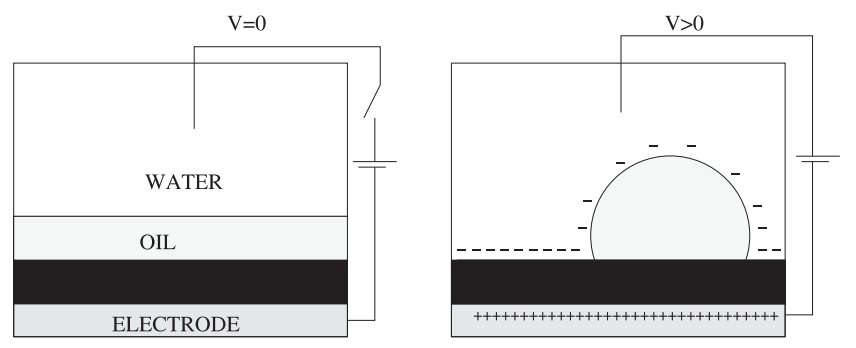

FIG. 2. Sketch of an optical switch.

advantage of the observation that stable films of colored oil sandwiched between water and hydrophobic insulators immediately contract to droplets when an external electric field is applied.

Models for electrowetting include a description for the movement of the liquid droplet. The classical formulation leads to a free boundary problem for the interface between the droplet and the ambient fluid. It is often convenient to approximate such problems by a phase-field model. This approximation has been established by Allen and Cahn [2] in connection with the kinetics of melted $\mathrm{Fe}-\mathrm{Al}$ alloys; it has been proved rigorously in many subsequent works and extended to some other rather different contexts. Phase-field models are typically derived by a gradient flow for a free energy functional of Ginzburg-Landau type that models the properties of the interface. Using the standard $L^{2}$ metric in the gradient flow gives models for non-conserved phases that have the form of non-linear parabolic equations of second order; these models are used to describe phase transitions. If the volume of the different phases is conserved it is advantageous to use the $H^{-1}$ metric in the gradient flow, which leads to a fourth order parabolic equation, the so called Cahn-Hilliard model [8]. The corresponding models are appropriate for phase separation and for free surface problems in fluids. Phase-field models have several advantages. Substitution of the equation for the movement of the free boundary by a partial differential equation simplifies the numerical solution and provides an easy way to handle topological changes in the domains occupied by each phase. It is, moreover, possible to include properties of the interface as e.g. surface tension into the model rather easily by choosing suitable constitutive functions. The idea of coupling interface problems in fluid mechanics with phase-field models has been explored only recently. The main idea consists in obtaining accurate representations of surface tension forces, acting on interfaces between fluids, in terms of the phase-field (see [24], [3] and the references therein). A mathematical proof of global (in time) existence of weak solutions of a particular model has been provided in [14], [15] (see also [1] for existence results for two-phase models with general densities). Another remarkable result of [14], [15] is the proof of convergence of finite element discretizations of the original model and of both phase-field models and their discretizations, in a suitable sense, to the original sharp interface problem. Additional difficulties arise if also the motion of contact lines is to be modeled, where liquid interfaces meet with a solid substrate. In [31], versions of phase-field models adequate for such wetting phenomena are proposed. Numerical experiments indicate that these approaches match quite accurately with molecular dynamics simulations.

In this paper we shall proceed a step further and study the problem in which the motion of interfaces is affected by the electric field produced by electric charges moving in the bulk of the fluids. In this way we provide a method to simulate phenomena such as electrowetting in the same spirit as in the above mentioned papers. 
A different approach to model aspects of electrowetting has been pursued by Lu et al. [25]. They propose a diffuse interface model—based on degenerate Cahn-Hilliard-dynamics—-for a liquid drop in a Hele-Shaw geometry. A comparison of numerical simulations and physical experiments shows good agreement with respect to shape dynamics and topology changes.

In the earlier paper [37], Walker and Shapiro studied also electrowetting in a Hele-Shaw cell. They combine level-set methods with Darcy's law to simulate droplet splitting and other microfluidical applications. The coupling with the electric field is performed by an explicit prescription of contact angles which are obtained from previous studies in the stationary case. By comparison with experiment, it becomes evident that models based only on the Lippmann theory lead qualitatively to numerical results being at variance with experimental data. In contrast, when also contact angle saturation effects are included, the matching between experiment and simulation is considerably improved.

Let us give the outline of the paper. In Section 2, the model is derived. First, we introduce the relevant energies-fluid-fluid as well as fluid-solid surface energies, electrostatic energy, kinetic energy and the free energy associated with charge distributions. Using Onsager's variational principle combined with conservation laws, we obtain a coupled pde system (see system (3.1p) for phase-field, velocity field, charge distribution and electrostatic potential. It is worth mentioning that the phase-field equation is subject to non-standard boundary conditions relating with each other a nonlinear term and derivatives with respect to time and to the normal direction. Recently, dynamical boundary conditions for the Cahn-Hilliard equation have been discussed in [32], with different techniques than in the present paper. Section 3 is devoted to the analysis of the model. In space dimension $d=2$ we obtain existence results for both the cases of degenerate and of non-degenerate conductivities. Due to a lack of regularity, in three space dimensions the case of degenerate conductivities can so far be treated only under the additional assumption of phase-independent dielectric parameters. In Section 4, we sketch the changes necessary to obtain related results in the case of negligible acceleration terms. Finally, in Section 5 some first numerical examples are presented to underline the applicability of our model. In particular, the numerical results are in accordance with the aforementioned results by Mugele and Bührle on the local persistence of the Young angle.

We finish the introduction with some remarks on notation. We use the usual notation for Lebesgue and Sobolev spaces as well as for derivatives with respect to space and time. We write $\Omega$ for the spatial domain in which the two fluids are contained. By $\Omega^{*}$, we denote a larger spatial domain containing $\Omega$ which also takes effects of the isolating wall into account. Therefore, phase field, velocity field, and charge density are only defined on $\Omega$, whereas the electric field has to be considered on $\Omega^{*}$. We often write $I$ for the time interval $(0, T)$, and we also abbreviate $\Omega \times I$ by $\Omega_{T}$. Here, of course, other combinations of domain and end-point in time are possible and will be used throughout the paper. Vector fields will be denoted in bold face, and we write $\mathcal{U}, \mathcal{V}, \mathcal{W}$ for the spaces $H_{0}^{1}\left(\Omega^{*}\right),\left\{\mathbf{v} \in\left(H_{0}^{1}(\Omega)\right)^{d} \mid \nabla \cdot \mathbf{v}=0\right\}$, and $H^{1}(\Omega)$, respectively. Tangential components of vector fields will be indicated by an index $\tau$, and we write $\dot{\rho}=\rho_{t}+\mathbf{v} \cdot \nabla \rho$ for the material time derivative. Generically, $\langle\cdot, \cdot\rangle$ denotes the $L^{2}$ scalar product, other dual pairings will be defined when needed.

\section{The model}

Our model is derived from basic principles in thermodynamics of irreversible processes near equilibrium. A related approach, in the simpler setting of a droplet spreading over a flat surface and confined to quasi-stationary motion, has been explored in [31]. In order to formulate the desired phase-field model, we introduce first the phase-field $\phi$ with the property that values of $\phi=+1$ or 
$\phi=-1$ correspond to the liquid droplet or the ambient fluid, respectively. Since the processes to be considered will take place at a temperature that we assume to be fixed, the natural thermodynamical quantity to describe these transformations is the so called free energy. Surface energies are included into phase-field models by a contribution to the free energy of the type (see $[8]$ for instance)

$$
F_{\phi}=\gamma_{0} \int_{\Omega}\left(\frac{\delta}{2}|\nabla \phi|^{2}+\frac{1}{\delta} W(\phi)\right)
$$

where $\Omega$ is the domain occupied by both the droplet and ambient fluid, $\gamma_{0}$ is the surface tension, $\delta$ is a small positive parameter and $W(\phi)$ is a double-well potential; for simplicity of presentation it will be defined as $W(\phi)=\left(1-\phi^{2}\right)^{2}$. In the present case, where our fluid is in contact with solid surfaces at $\partial \Omega$, we have to introduce another term due to the interfacial energy at the fluid-solid interface,

$$
F_{f s}=\int_{\partial \Omega} \gamma_{f s}(\phi)
$$

Here, $\gamma_{f s}(\phi)$ is—up to a constant-a smooth function which interpolates the values of the fluid-

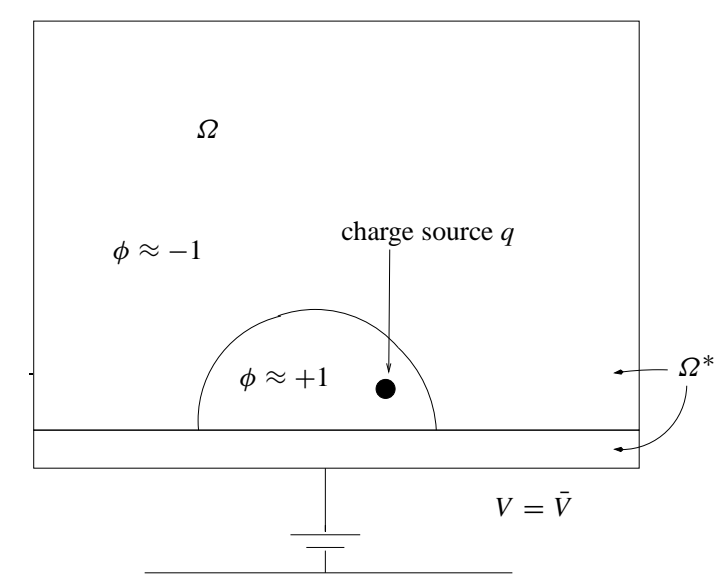

FIG. 3. Sketch of the physical setting: The two liquids are contained in $\Omega . \Omega^{*}$ is a larger domain which takes the dielectric layer into account.

solid interface energies of the different fluids. We may take, for instance,

$$
\gamma_{f s}(\phi)=\frac{\gamma_{f s}^{(1)}-\gamma_{f s}^{(2)}}{2} \sin \frac{\pi \phi}{2} .
$$

The numbers $\gamma_{f s}^{(i)}, i=1,2$, stand for the surface energies between the solid and the $i$-th fluid.

The electric field adds to the total free energy a contribution (see [22])

$$
F_{\mathbf{D}}=\frac{1}{2} \int_{\Omega} \frac{|\mathbf{D}|^{2}}{\varepsilon(\phi)}
$$

where $\varepsilon(\phi)$ is the dielectric constant and $\mathbf{D}=\varepsilon(\phi) \mathbf{E}$ the (electric) displacement vector. We neglect the effects of induced magnetic fields in our model, so that the electric field is curl-free (by Maxwell 
equations), and we can express it in terms of the electrostatic potential by means of the relation $\mathbf{E}=-\nabla V$.

The inertia of the fluid would add a kinetic energy to the free energy of the form

$$
F_{\mathbf{v}}=\frac{1}{2} \int_{\Omega} d(\phi)|\mathbf{v}|^{2}
$$

where $d(\phi)$ is the density of the fluid material. We expect the effects caused by relative variations in density to be marginal in electrowetting. Hence, we will assume the density to be independent of $\phi$ and to be equal to 1 .

Additionally we include a term in the free energy due to space distribution of charges $\rho$ of the form

$$
F_{\rho}=\frac{\lambda}{2} \int_{\Omega} \rho^{2}
$$

where $\lambda \ll 1$. This term will serve as a useful regularization for our model, by transforming surface densities of charges that typically arise in static electricity to thin volume layers that are better suited for combination with phase-field models. In fact, this term will lead to a diffusive flux of charges, which combined with the flux associated to the term $(2.1)$ will make the thickness of the charge layer typically of the order of $\sqrt{\lambda \varepsilon(0)}$, setting an upper bound for the grid size of the numerical methods necessary to resolve such a layer.

The total free energy is then

$$
F=F_{\phi}+F_{f s}+F_{\mathbf{D}}+F_{\mathbf{v}}+F_{\rho}
$$

The phase-field $\phi$ will evolve in time following a convective Cahn-Hilliard equation

$$
\frac{\partial \phi}{\partial t}+\nabla \cdot(\phi \mathbf{v})=-\nabla \cdot \mathbf{J}_{\phi}
$$

where $\mathbf{J}_{\phi}$ and $\mathbf{v}$ denote flux and velocity fields, respectively.

The displacement vector $\mathbf{D}$ will evolve according to Maxwell's equations (cf. [19]). Since fluid and charge velocities are small in comparison to the velocity of light, magnetic effects due to the motion of charges will be neglected, which leaves us with the equation

$$
\mathbf{D}_{t}=-\rho \mathbf{v}-\mathbf{J}_{\mathbf{D}}
$$

where the subscript $t$ denotes the partial time derivative. The flux is given by the sum of convective motion of charges and a flux $\mathbf{J}_{\mathbf{D}}$ which is a combination of conduction across the medium following Ohm's law and diffusive flux of charges.

A second equation from Maxwell's system is

$$
\nabla \cdot \mathbf{D}=\rho,
$$

which we assume to hold in a larger domain $\Omega^{*}$ such that $\Omega \subset \Omega^{*}$ (with $\rho=0$ at $\Omega^{*} \backslash \Omega$ ). Such a construction is needed as we have to take into account dielectric layers which separate the liquids from the counter electrodes. Hence, the boundary conditions on $V$ will be imposed at $\partial \Omega^{*}$, and the presence of different materials in $\Omega$ and in $\Omega^{*} \backslash \Omega$ will be reflected by a dependence of the dielectric constant $\varepsilon$ on the spatial coordinates and the phase-field. 
Taking the divergence in (2.3) and the time derivative of (2.4), the following evolution equation for $\rho$ can easily be derived:

$$
\frac{\partial \rho}{\partial t}=-\nabla \cdot(\rho \mathbf{v})-\nabla \cdot \mathbf{J}_{\mathbf{D}}
$$

Finally, the velocity field $\mathbf{v}$ satisfies the Navier-Stokes equations

$$
\begin{aligned}
\frac{\partial \mathbf{v}}{\partial t}+\mathbf{v} \cdot \nabla \mathbf{v} & =\nabla \cdot \Pi+\mathbf{F}, \\
\nabla \cdot \mathbf{v} & =0,
\end{aligned}
$$

where $\mathbf{F}$ is the field of external forces exerted on the fluids and

$$
\Pi=\eta(\phi) \mathbf{T}(\mathbf{v})-p I
$$

denotes the stress tensor, with viscosity $\eta=\eta(\phi)$, strain velocity tensor $\mathbf{T}(\mathbf{v})=\frac{1}{2}\left(\nabla \mathbf{v}+\nabla \mathbf{v}^{t}\right)$ and pressure $p$.

We assume the normal velocity $\mathbf{v} \cdot \mathbf{n}$ and the fluxes $\mathbf{J}_{\phi} \cdot \mathbf{n}$ and $\mathbf{J}_{\mathbf{D}} \cdot \mathbf{n}$ to vanish at the boundary of $\Omega$. Then one can compute the variation of $F=F(\phi, \mathbf{D}, \mathbf{v}, \rho)$. Let us begin with the variation with respect to $\phi$,

$$
\delta F=\int_{\Omega} \mu \delta \phi+\int_{\partial \Omega} L \delta \phi,
$$

where we write briefly

$$
\begin{aligned}
\mu & :=\gamma_{0}\left(-\delta \Delta \phi+\frac{1}{\delta} W^{\prime}(\phi)\right)-\frac{\varepsilon^{\prime}(\phi)}{2 \varepsilon^{2}(\phi)}|\mathbf{D}|^{2}, \\
L & :=\gamma_{f s}^{\prime}(\phi)+\gamma_{0} \delta \frac{\partial \phi}{\partial n}
\end{aligned}
$$

for the chemical potential in the bulk and at the solid surface.

Altogether, for the variation of $F$ we obtain

$$
\delta F=\int_{\Omega} \mathbf{v} \cdot \delta \mathbf{v}+\int_{\Omega} \mu \delta \phi+\int_{\partial \Omega} L \delta \phi+\int_{\Omega} \mathbf{E} \cdot \delta \mathbf{D}+\lambda \int_{\Omega} \rho \delta \rho .
$$

We write the variation of $F$ with respect to time by integrating the local variations of the free energy over the whole domain $\Omega$,

$$
\frac{\mathrm{d} F}{\mathrm{~d} t}=\int_{\Omega} \mathbf{v} \cdot \frac{\partial \mathbf{v}}{\partial t}+\int_{\Omega} \mu \frac{\partial \phi}{\partial t}+\int_{\partial \Omega} L \frac{\partial \phi}{\partial t}+\int_{\Omega} \mathbf{E} \cdot \frac{\partial \mathbf{D}}{\partial t}+\lambda \int_{\Omega} \rho \frac{\partial \rho}{\partial t} .
$$

Integration by parts of (2.9) and using 2.2], 2.5 - 2.7) and the fact that the normal component of the velocity and the fluxes vanish at $\partial \Omega$ allow us to conclude

$$
\begin{aligned}
\frac{\mathrm{d} F}{\mathrm{~d} t}= & \int_{\partial \Omega} \mathbf{n} \cdot\left(\Pi \mathbf{v}_{\tau}\right)-\int_{\Omega} \Pi: \nabla \mathbf{v}+\int_{\Omega} \mathbf{v} \cdot \mathbf{F} \\
& +\int_{\Omega} \mu\left(-\nabla \cdot(\phi \mathbf{v})-\nabla \cdot \mathbf{J}_{\phi}\right)+\int_{\partial \Omega} L\left(\dot{\phi}-\mathbf{v}_{\tau} \cdot(\nabla \phi)_{\tau}\right) \\
& +\int_{\Omega} \mathbf{E} \cdot\left(-\rho \mathbf{v}-\mathbf{J}_{\mathbf{D}}\right)+\lambda \int_{\Omega} \rho\left(-\nabla \cdot(\rho \mathbf{v})-\nabla \cdot \mathbf{J}_{\mathbf{D}}\right) .
\end{aligned}
$$


Here, $\dot{\phi}$ is a short notation for the material derivative, and the subscript $\tau$ denotes the tangential component of a vector field defined on $\partial \Omega$. Identifying the rate of change of the mechanical work with

$$
\frac{\mathrm{d} W}{\mathrm{~d} t}=\int_{\Omega} \mathbf{v} \cdot \mathbf{F}-\int_{\Omega} \mu \mathbf{v} \cdot \nabla \phi-\int_{\Omega} \rho \mathbf{v} \cdot \mathbf{E}+\lambda \int_{\Omega} \rho \mathbf{v} \cdot \nabla \rho
$$

and writing

$$
\begin{aligned}
\frac{\mathrm{d} S}{\mathrm{~d} t}=-\frac{1}{T}\left(-\int_{\Omega} \Pi: \nabla \mathbf{v}-\int_{\Omega} \mathbf{E} \cdot \mathbf{J}_{\mathbf{D}}\right. & +\lambda \int_{\Omega} \nabla \rho \cdot \mathbf{J}_{\mathbf{D}}+\int_{\Omega} \nabla \mu \cdot \mathbf{J}_{\phi} \\
& \left.+\int_{\partial \Omega} L \dot{\phi}+\int_{\partial \Omega} \mathbf{n} \cdot\left(\Pi \mathbf{v}_{\tau}\right)-\int_{\partial \Omega} L \mathbf{v}_{\tau}(\nabla \phi)_{\tau}\right)
\end{aligned}
$$

for the change of entropy in time, we find that equation 2.10 is in accordance with the well known thermodynamic equation

$$
\frac{\mathrm{d} F}{\mathrm{~d} t}=\frac{\mathrm{d} W}{\mathrm{~d} t}-T \frac{\mathrm{d} S}{\mathrm{~d} t} .
$$

Since no external forces are included into our model, we conclude that no net work is being produced by or in the bulk. Therefore, treating $\mathbf{v}$ as arbitrary in the expression 2.11 for the variation of the free energy, we find that such a condition on the mechanical work necessarily leads to the following expression for the force:

$$
\mathbf{F}=\mu \nabla \phi+\rho \mathbf{E}-\nabla\left(\frac{\lambda}{2} \rho^{2}\right) .
$$

The Navier-Stokes system 2.6, 2.7) can then be written, after redefining $p$ by adding $(\lambda / 2) \rho^{2}$, as

$$
\frac{\partial \mathbf{v}}{\partial t}+\mathbf{v} \cdot \nabla \mathbf{v}=\nabla \cdot \Pi+\mathbf{F}_{s t}+\mathbf{F}_{p}+\mathbf{F}_{e}
$$

where

$$
\begin{aligned}
\mathbf{F}_{s t} & =\gamma_{0}\left(-\delta \Delta \phi+\frac{1}{\delta} W^{\prime}(\phi)\right) \nabla \phi \\
\mathbf{F}_{p} & =-\frac{1}{2} \varepsilon^{\prime}(\phi)|\mathbf{E}|^{2} \nabla \phi, \\
\mathbf{F}_{e} & =\rho \mathbf{E}
\end{aligned}
$$

correspond to surface tension, Maxwell stress and electrostatic forces, respectively.

A variational principle by Onsager (see [29]) establishes that, in absence of mechanical work, the variation with respect to the fluxes of the difference between the rate of change of the entropy and the energy dissipation has to cancel during the evolution of the system. This principle, applied to our case where mechanical work is actually produced (see [31] where a similar approach is introduced), leads to

where the fluxes are

$$
\delta_{\mathbf{J}}(\dot{F}(\mathbf{J})+\Phi(\mathbf{J}, \mathbf{J}))=0
$$

$$
\mathbf{J}=\left(\Pi, \dot{\phi}, \mathbf{J}_{\phi}, \mathbf{J}_{\mathbf{D}}, \mathbf{v}_{\tau}\right)
$$

The usual expression for the dissipation function is the sum of quadratic terms in the fluxes with phenomenological parameters $M(\phi), \alpha^{-1}, K(\phi), \eta(\phi)$ and $\beta$ :

$$
\Phi(\mathbf{J}, \mathbf{J})=\int_{\Omega} \frac{\left|\mathbf{J}_{\phi}\right|^{2}}{2 M(\phi)}+\int_{\partial \Omega} \frac{\dot{\phi}^{2}}{2 \alpha^{-1}}+\int_{\Omega} \frac{\left|\mathbf{J}_{\mathbf{D}}\right|^{2}}{2 K(\phi)}+\int_{\Omega} \frac{|\Pi|^{2}}{2 \eta(\phi)}+\int_{\partial \Omega} \frac{\beta}{2}\left|\mathbf{v}_{\tau}\right|^{2}
$$


and it equals half the total rate of energy dissipation. For the dissipation associated to the viscous flow we have used (cf. [18, Chapter XII])

$$
\int_{\Omega} \frac{|\Pi|^{2}}{2 \eta(\phi)}
$$

The classical boundary condition for fluids in contact with solid surfaces is the no-slip boundary condition, $\mathbf{v}=0$ at $\partial \Omega$. This would cancel out the last term on the right-hand side of (2.16). When one imposes that only the normal component of the velocity field vanishes, this term describes, for $\beta>0$, some viscous dissipation caused by the tangential movement of the fluid at the boundary; it leads to the so-called Navier boundary condition (see [28], [31]). This condition has been proposed as a means to solve the contact line paradox, that is, the development of unbounded energy dissipations due to the motion of contact lines over the substrate, in the neighborhood of the contact line. Nevertheless, numerical evidence (see [3]) suggests that phase-field models introduce a regularization sufficient to solve the contact line paradox and to allow for moving contact lines. In this spirit, in the present work we shall consider the no-slip boundary condition, but we will also give the equations in the case of partial slip for the sake of completeness.

Solving the variational problem 2.15 , we obtain the fluxes

$$
\begin{aligned}
\mathbf{J}_{\phi} & =-M(\phi) \nabla \mu \quad \text { in } \Omega, \\
\mathbf{J}_{\mathbf{D}} & =K(\phi)(\mathbf{E}-\lambda \nabla \rho) \quad \text { in } \Omega, \\
\alpha \dot{\phi} & =-L(\phi)=-\left(\gamma_{f s}^{\prime}(\phi)+\gamma_{0} \delta \frac{\partial \phi}{\partial n}\right) \quad \text { on } \partial \Omega,
\end{aligned}
$$

together with the Navier condition

$$
\beta \mathbf{v}_{\tau}=-\eta(\phi)(\mathbf{T}(\mathbf{v}) \mathbf{n})_{\tau}+L(\phi)(\nabla \phi)_{\tau} .
$$

Recalling 2.2, we observe that the phase-field $\phi$ evolves according to Cahn-Hilliard dynamics

$$
\frac{\partial \phi}{\partial t}+\nabla \cdot(\phi \mathbf{v})-\nabla \cdot(M(\phi) \nabla \mu)=0 .
$$

Equation (2.18) allows us to identify $\mathbf{J}_{\mathbf{D}}$ as the flux due to the sum of a conductive flux described by Ohm's law, $\mathbf{J}_{\mathbf{D}}^{(1)}=K(\phi) \mathbf{E}$ with $K(\phi)$ the electric conductivity, and a diffusive flux for the electric charges $\mathbf{J}_{\mathbf{D}}^{(2)}=-D(\phi) \nabla \rho$ where the diffusion coefficient $D(\phi)$ is proportional to the conductivity with a proportionality constant $\lambda$. The diffusive flux will always be considered small in comparison to the conductive flux. In fact, we shall take

$$
\frac{D(\phi)}{K(\phi)}=\lambda \ll 1
$$

In the literature (cf. [34]), the case of electrolytes (negative or positive ions) moving in a dielectric medium is also considered so that the conductive flux is not merely $K(\phi) \mathbf{E}$ but $\nu(\phi) \rho \mathbf{E}$ with the charge mobility $v(\phi)$ in the medium. In this case, the relation between mobility and diffusivity, $v(\phi) / D(\phi)$ is known to be constant, once in statistical equilibrium, and according to Einstein's relation, inversely proportional to temperature. Relation 2.21 may be considered a reminiscence 
of this fact, but for our purposes it is merely a technical device that will make our calculations simpler without changing the models in a significant manner.

Notice that the resulting equations for the fluxes 2.17)-2.19) are linear combinations of the thermodynamic forces (in the notation introduced by Onsager in his study of thermodynamical processes close to equilibrium, see [18]) $-\nabla \mu, \mathbf{E}=-\nabla V,-\nabla \rho, \nabla \mathbf{v},-L$. In the same spirit, the entropy production (2.12) may be viewed as $T^{-1}$ times the sum of the products of the fluxes $\mathbf{J}_{i}$ in $\mathbf{J}=\left(\Pi, \dot{\phi}, \mathbf{J}_{\phi}, \mathbf{J}_{\mathbf{D}}\right)$ and thermodynamic forces $\mathbf{X}_{i}$ in $\mathbf{X}=(\nabla \mathbf{v},-L,-\nabla \mu, E-\lambda \nabla \rho)$,

$$
\frac{\mathrm{d} S}{\mathrm{~d} t}=\frac{1}{T} \sum \mathbf{J}_{i} \cdot \mathbf{X}_{i}
$$

as established in the celebrated theory of irreversible processes introduced by Onsager in 1931 (see [29] and [18]).

To summarize, we are left with a system consisting of $(2.2)$ with $\mathbf{J}_{\phi}$ given by $(2.17)$ and $\mu$ by 2.8) with boundary conditions $\mathbf{J}_{\phi} \cdot \mathbf{n}=0$ and

$$
\begin{aligned}
& \alpha \dot{\phi}=-L(\phi)=-\left(\gamma_{f_{s}}^{\prime}(\phi)+\gamma_{0} \delta \frac{\partial \phi}{\partial n}\right) \quad \text { on } \partial \Omega, \\
& \frac{\partial \mu}{\partial \mathbf{n}}=0 \quad \text { on } \partial \Omega,
\end{aligned}
$$

equations (2.4) and 2.5) with $\mathbf{D}=\varepsilon(\phi) \mathbf{E}=-\varepsilon(\phi) \nabla V$ and boundary conditions

$$
V=\bar{V} \quad \text { at } \partial \Omega^{*}, \quad \mathbf{J}_{\mathbf{D}} \cdot \mathbf{n}=0 \quad \text { at } \partial \Omega,
$$

and equation 2.13 with forces given by 2.14 and boundary conditions

$$
\begin{aligned}
\mathbf{v} \cdot \mathbf{n} & =0 \quad \text { on } \partial \Omega, \\
\mathbf{v}_{\tau}=0 \quad \text { or } \quad \beta \mathbf{v}_{\tau} & =-\eta(\phi)(\mathbf{T}(\mathbf{v}) \mathbf{n})_{\tau}+L(\nabla \phi)_{\tau} \quad \text { on } \partial \Omega .
\end{aligned}
$$

In the case of negligible acceleration of the fluid, we may replace the Navier-Stokes system by a quasi-stationary Stokes system. The deduction of the corresponding system from thermodynamic principles would follow essentially the same path except for the absence of $F_{\mathbf{v}}$ in the total free energy. The terms $\int_{\Omega} \mathbf{v} \cdot \mathbf{F}$ and $\int_{\Omega} \Pi: \nabla \mathbf{v}$ would still be present in the variation of total work and entropy respectively since they are balanced through the Stokes system:

$$
\nabla \cdot \Pi+\mathbf{F}=0
$$

Hence the expressions for all the different forces and fluxes will be exactly the same in this case.

Finally, it might happen in the context of electrowetting that the potential has to be kept constant between two electrodes, which can be achieved by placing an appropriate amount of charge in them at a constant potential. Assume an amount of charges $q(x, t)$ per unit time and unit volume is placed at the point $x$ at time $t$. We can think for instance of the charge being placed at $\Gamma_{0}$, denoting the region occupied by an electrode inside the droplet. Therefore, the equation for $\rho$ will be, instead of (2.5),

$$
\frac{\partial \rho}{\partial t}+\nabla \cdot(\rho \mathbf{v})+\nabla \cdot(K(\phi)(\mathbf{E}-\lambda \nabla \rho))=q
$$

with the boundary condition

$$
K(\phi)(\mathbf{E}-\lambda \nabla \rho) \cdot \mathbf{n}=0 \quad \text { at } \partial \Omega .
$$




\section{Existence of weak solutions}

In this section we present results on the existence of solutions to our model. This is done in two steps: first we consider a model with non-degenerate electric conductivity, where $K(\phi) \geqslant K_{-}>0$ for every value of $\phi$. From the physical point of view, this model is rather unrealistic as it allows for diffusive and field-induced charge transport also outside the conductive phase. Here, it only serves mathematically for approximation purposes. In Subsection 3.4. we shall analyze the case of a degenerate conductivity $K(-1)=0$-in two space dimensions without any further restrictions, in three space dimensions under the assumption of a phase-independent dielectric parameter.

Assuming constant unit mass density $(d \equiv 1)$, the model consists of the following differential equations:

$$
\begin{aligned}
\mathbf{v}_{t}+(\mathbf{v} \cdot \nabla) \mathbf{v}-\nabla \cdot(\eta(\phi) \mathbf{T}(\mathbf{v}))+\nabla p-\mu \nabla \phi+\rho \nabla V=0 & \text { in } \Omega_{T}, \\
\nabla \cdot \mathbf{v}=0 & \text { in } \Omega_{T}, \\
\rho_{t}+\mathbf{v} \cdot \nabla \rho-\nabla \cdot(K(\phi) \nabla(V+\lambda \rho))=q & \text { in } \Omega_{T}, \\
\phi_{t}+\mathbf{v} \cdot \nabla \phi-\nabla \cdot(M(\phi) \nabla \mu)=0 & \text { in } \Omega_{T}, \\
-\nabla \cdot(\bar{\varepsilon}[\phi] \nabla V) & = \begin{cases}\rho & \text { in } \Omega \forall t \in(0, T), \\
0 & \text { in } \Omega^{*} \backslash \Omega \forall t \in(0, T) .\end{cases}
\end{aligned}
$$

Here, the velocity strain tensor $\mathbf{T}$ and the chemical potential $\mu$ are given by

$$
\begin{aligned}
\mathbf{T}(\mathbf{v}) & =\frac{1}{2}\left(\nabla \mathbf{v}+(\nabla \mathbf{v})^{t}\right), \\
\mu & =\gamma_{0}\left(-\delta \Delta \phi+\frac{1}{\delta} W^{\prime}(\phi)\right)-\frac{1}{2} \varepsilon^{\prime}(\phi)|\nabla V|^{2} .
\end{aligned}
$$

We further assume $\gamma_{0} \equiv 1$ without loss of generality. As boundary conditions, we have

$$
\begin{aligned}
\mathbf{v} & =0, \\
K(\phi) \nabla(V+\lambda \rho) \cdot \mathbf{n} & =0, \\
\frac{\partial}{\partial \mathbf{n}} \mu & =0, \\
\gamma_{0} \delta \frac{\partial}{\partial \mathbf{n}} \phi & =-\gamma_{f s}^{\prime}(\phi)-\alpha \phi_{t}
\end{aligned}
$$

on $\partial \Omega \times(0, T)$ and

$$
V=\bar{V} \quad \text { on } \partial \Omega^{*} \times(0, T) .
$$

Let us specify our assumptions on domains and data.

(H0) The domains $\Omega \subset \Omega^{*} \subset \mathbb{R}^{d}, d=2,3$, are either bounded with boundary of class $C^{1,1}$ or bounded and convex with Lipschitz boundary.

(H1) The dielectric parameter $\varepsilon(\cdot) \in C^{1,1}(\mathbb{R})$ satisfies $0<\varepsilon_{-} \leqslant \varepsilon(\phi) \leqslant \varepsilon_{+}<\infty$ for all $\phi \in \mathbb{R}$ and is monotone with $\operatorname{supp}\left(\varepsilon^{\prime}\right) \subset[-1,1]$. On $\Omega_{T}^{*}$, we will also consider the function

$$
\bar{\varepsilon}[\phi](t, x):= \begin{cases}\varepsilon(\phi(t, x)) & \text { if } x \in \bar{\Omega}, \\ \varepsilon_{*}(x) & \text { if } x \in \bar{\Omega}^{*} \backslash \bar{\Omega} .\end{cases}
$$

Here, $\varepsilon_{*} \in L^{\infty}\left(\Omega^{*} \backslash \Omega\right)$ is bounded from below by a positive parameter. 
(H2) The mobility $M(\cdot) \in C^{1,1}(\mathbb{R}) \cap L^{\infty}(\mathbb{R})$ is bounded from below by a positive constant $M_{-}$.

(H3) The interfacial energy $\gamma_{f s} \in C^{1}(\mathbb{R})$ and the viscosity function $\eta \in C^{1,1}(\mathbb{R})$ are monotone on $[-1,1]$ with $\operatorname{supp}\left(\gamma_{f s}^{\prime}\right) \subset[-1,1]$.

(H4) The conductivity function $K(\cdot) \in C^{1}(\mathbb{R})$ satisfies $0 \leqslant K(\cdot) \leqslant K_{+}<\infty$ and is monotone increasing with $\operatorname{supp}\left(K^{\prime}\right) \subset[-1,1]$.

(H4-bis) Special case of non-degenerate conductivities: there is a positive constant $K_{-}$such that $K(\cdot) \geqslant K_{-}$on $\mathbb{R}$

(H5) $\bar{V} \in L^{\infty}\left(I ; W^{1, r}\left(\Omega^{*}\right)\right) \cap H^{1}\left(I ; H^{1}\left(\Omega^{*}\right)\right)$ for an exponent $r>2$ and arbitrary time intervals $I$.

Note that (H1), (H3), (H4), and (H4-bis) put into simple mathematical terms the physical fact that we are dealing with a mixture of two different liquids and therefore the physical quantities of dielectricity, interfacial energy, viscosity, and conductivity depend on the phase-field. (H2) and (H5) are merely technical assumptions.

\subsection{Weak formulation}

We introduce the function spaces

$$
\mathcal{V}:=\left\{\mathbf{v} \in\left(H_{0}^{1}(\Omega)\right)^{d} \mid \nabla \cdot \mathbf{v}=0\right\}
$$

for the velocity field,

$$
\mathcal{W}:=H^{1}(\Omega)
$$

for the functions $\rho, \phi, \mu$, and

$$
\mathcal{U}:=H_{0}^{1}\left(\Omega^{*}\right)
$$

for the potential excess $V-\bar{V}$. Moreover, we will sometimes use the notation $\mathcal{S}_{d}^{\prime}$ for the dual space of $\mathcal{S}_{d}:=\left\{\mathbf{v} \in\left(W_{0}^{1, d}(\Omega)\right)^{d} \mid \nabla \cdot \mathbf{v}=0\right\}, d \in\{2,3\}$. Of course, $\mathcal{S}_{2}^{\prime}=\mathcal{V}^{\prime}$.

The weak formulation of the problem is as follows.

Definition 3.1 Let $p$ satisfy $2<p<\infty$ if $d=2$, and $p=3$ if $d=3$. Assume the source term $q$ to be an element of $L^{2}\left(\Omega_{T}\right)$. For given initial data $\mathbf{v}_{0} \in \mathcal{V}, \phi_{0} \in \mathcal{W}$, and $\rho_{0} \in L^{2}(\Omega)$, we call a quintuple $(\rho, \phi, \mathbf{v}, \mu, V)$ with

$$
\begin{aligned}
\rho & \in L^{\infty}\left(I ; L^{2}(\Omega)\right) \cap H^{1}\left(I ; W^{1, p}(\Omega)^{\prime}\right), \\
\phi & \in L^{\infty}\left(I ; H^{1}(\Omega)\right) \cap H^{1}\left(I ; H^{1}(\Omega)^{\prime}\right) \cap H^{1}\left(I ; L^{2}(\partial \Omega)\right), \\
\mathbf{v} & \in L^{\infty}\left(I ; L^{2}(\Omega)\right) \cap L^{2}(I ; \mathcal{V}) \cap W^{1,6 / 5}\left(I ; \mathcal{S}_{d}^{\prime}\right), \\
\mu & \in L^{2}\left(I ; H^{1}(\Omega)\right), \\
V-\bar{V} & \in L^{\infty}\left(I ; H^{1}(\Omega)\right)
\end{aligned}
$$

a weak solution of 3.1-3.4 if the following hold:

$$
\begin{aligned}
& \int_{0}^{T}\left\langle\mathbf{v}_{t}, \mathbf{w}\right\rangle_{1}+\int_{\Omega_{T}}((\mathbf{v} \cdot \nabla) \mathbf{v} \cdot \mathbf{w}+\eta(\phi) \mathbf{T}(\mathbf{v}): \mathbf{T}(\mathbf{w}))+\int_{\Omega_{T}}(\rho \nabla V-\mu \nabla \phi) \cdot \mathbf{w}=0, \\
& \int_{0}^{T}\left\langle\rho_{t}, \psi_{1}\right\rangle_{2}+\int_{\Omega_{T}}(K(\phi) \nabla(V+\lambda \rho)-\rho \mathbf{v}) \cdot \nabla \psi_{1}=\int_{\Omega_{T}} q \psi_{1},
\end{aligned}
$$




$$
\begin{aligned}
& \int_{0}^{T}\left\langle\phi_{t}, \psi_{2}\right\rangle_{3}+\int_{\Omega_{T}}\left(\mathbf{v} \cdot \nabla \phi \psi_{2}+M(\phi) \nabla \mu \cdot \nabla \psi_{2}\right)=0 \\
& \int_{\Omega_{T}} \mu \psi_{3}=\int_{\Omega_{T}}\left(\delta \nabla \phi \cdot \nabla \psi_{3}+\frac{1}{\delta} W^{\prime}(\phi) \psi_{3}-\frac{1}{2} \varepsilon^{\prime}(\phi)|\nabla V|^{2} \psi_{3}\right)+\int_{0}^{T} \int_{\partial \Omega}\left(\alpha \phi_{t}+\gamma_{f s}^{\prime}(\phi)\right) \psi_{3} \\
& \int_{\Omega_{T}^{*}} \bar{\varepsilon}[\phi] \nabla V \cdot \nabla \chi=\int_{\Omega_{T}} \rho \chi
\end{aligned}
$$

for every $\mathbf{w} \in L^{6}\left(I ; \mathcal{S}_{d}\right), \psi_{1} \in L^{2}\left(I ; W^{1, p}(\Omega)\right), \psi_{2} \in L^{2}\left(I ; H^{1}(\Omega)\right), \psi_{3} \in L^{2}\left(I ; H^{1}(\Omega)\right) \cap$ $L^{1}\left(I ; L^{\infty}(\Omega)\right)$, and $\chi \in L^{2}(I ; \mathcal{U})$. Here, $\langle\cdot, \cdot\rangle_{1},\langle\cdot, \cdot\rangle_{2}$ and $\langle\cdot, \cdot\rangle_{3}$ denote the dual pairings on the spaces $L^{6}\left(I ; \mathcal{S}_{d}\right), L^{2}\left(I ; W^{1, p}(\Omega)\right)$, and $L^{2}\left(I ; H^{1}(\Omega)\right)$, respectively.

\subsection{A formal energy estimate}

The key result for the subsequent analysis is an energy estimate which comes as a natural consequence of the derivation of the model. Taking $\mathbf{w}=\mathbf{v}$ in $(3.5 \mathrm{a}), \psi_{1}=\lambda \rho+V$ in $(3.5 \mathrm{~b})$, $\psi_{2}=\mu$ in $3.5 \mathrm{c}$ and $\psi_{3}=\phi_{t}$ in $3.5 \mathrm{~d}$, as well as differentiating the integrand of 3.5e with respect to time and choosing $\chi=V-\bar{V}$ as a test function gives after adding all relations

$$
\begin{aligned}
{\left[\int _ { \Omega } \left(\frac{1}{2}|\mathbf{v}|^{2}+\frac{\lambda}{2} \rho^{2}+\right.\right.} & \left.\frac{\delta}{2}|\nabla \phi|^{2}+\frac{1}{\delta} W(\phi)\right)+ \\
+\int_{\Omega_{T}}\left[\eta(\phi)|\mathbf{T}(\mathbf{v})|^{2}\right. & \left.+K(\phi)|\nabla(V+\lambda \rho)|^{2}+M(\phi)|\nabla \mu|^{2}\right]+\int_{0}^{T} \int_{\partial \Omega} \alpha\left|\phi_{t}\right|^{2} \\
= & \int_{\Omega_{T}} q\left(V+\left.\lambda V\right|^{2}+\int_{\partial \Omega} \gamma_{f s}(\phi)\right]_{0}^{T} \\
& -\int_{\Omega_{T}} \bar{\varepsilon}[\phi] \nabla V \partial_{t} \nabla \bar{V}-\left[\int_{\Omega} \rho \bar{V}\right]_{0}^{T}+\int_{\Omega_{T}} \rho \bar{V}_{t} .
\end{aligned}
$$

By standard absorption techniques, we find

$$
\begin{aligned}
\int_{\Omega} \rho^{2}(\cdot, T)+ & \int_{\Omega^{*}} \bar{\varepsilon}[\phi]|\nabla V|^{2}(\cdot, T) \\
\leqslant & R_{0}\left(\mathbf{v}_{0}, \rho_{0}, \phi_{0}\right)+C_{1}\left\{\int_{\Omega_{T}} q^{2}+\int_{\Omega_{T}}\left|\partial_{t} \nabla \bar{V}\right|^{2}+\int_{\Omega_{T}}\left|\bar{V}_{t}\right|^{2}+\sup _{t \in[0, T]} \int_{\Omega}|\bar{V}|^{2}\right\} \\
& +C_{2}\left\{\int_{\Omega_{T}} \rho^{2}+\int_{\Omega_{T}} \varepsilon(\phi)|\nabla V|^{2}\right\}
\end{aligned}
$$

with constants $C_{1}, C_{2}$ depending only on data and time $T$. In particular, $R_{0}$ is a non-negative term which collects the contributions of initial data. Applying Gronwall's lemma, we infer that

$$
\sup _{t \in[0, T]}\left\{\int_{\Omega} \rho^{2}(\cdot, t)+\int_{\Omega^{*}} \bar{\varepsilon}[\phi]|\nabla V|^{2}(\cdot, t)\right\}
$$


is bounded by a constant $C<\infty$ which only depends on data and time $T$. Inserting this in (3.6) and using Korn's inequality, we finally have

$$
\begin{gathered}
\|\rho\|_{L^{\infty}\left(I ; L^{2}(\Omega)\right)}+\|\phi\|_{L^{\infty}\left(I ; H^{1}(\Omega)\right)}+\|W(\phi)\|_{L^{\infty}\left(I ; L^{1}(\Omega)\right)}+\|V\|_{L^{\infty}\left(I ; H^{1}\left(\Omega^{*}\right)\right)}+\|\mathbf{v}\|_{L^{\infty}\left(I ; L^{2}(\Omega)\right)} \\
+\|\mathbf{v}\|_{L^{2}\left(I ; H^{1}(\Omega)\right)}+\left\|K(\phi)^{1 / 2} \nabla \rho\right\|_{L^{2}\left(\Omega_{T}\right)}+\|\nabla \mu\|_{L^{2}\left(\Omega_{T}\right)}+\left\|\phi_{t}\right\|_{L^{2}(\partial \Omega \times I)} \leqslant C
\end{gathered}
$$

with a constant $C$ that only depends on the given data and time $T$. This estimate also holds for the case of degenerate electric conductivity; the lower bound for $K(\phi)$ may be zero.

Estimate 3.7 and equations 3.5b, 3.5c imply

$$
\left\|\phi_{t}\right\|_{L^{2}\left(I ; H^{1}(\Omega)^{\prime}\right)}+\left\|\rho_{t}\right\|_{L^{2}\left(I ;\left(W^{1, p}(\Omega)\right)^{\prime}\right)} \leqslant C
$$

with

and arbitrary positive $v$.

$$
p= \begin{cases}2+v & \text { for } d=2 \\ 3 & \text { for } d=3\end{cases}
$$

\subsection{The case of non-degenerate electric conductivity}

The existence of a solution to system 3.5 with $K(\phi) \geqslant K_{-}>0$ is established by the FaedoGalerkin method. We use finite-dimensional subspaces

$$
\begin{aligned}
\mathcal{V}_{n}:=\operatorname{span}\left\{\mathbf{w}_{1}, \ldots, \mathbf{w}_{n}\right\} & \text { of } \mathcal{V}, \\
\mathcal{W}_{n}:=\operatorname{span}\left\{\psi_{1}, \ldots, \psi_{n}\right\} & \text { of } \mathcal{W}, \\
\mathcal{U}_{n}:=\operatorname{span}\left\{\chi_{1}, \ldots, \chi_{n}\right\} & \text { of } \mathcal{U}
\end{aligned}
$$

Here, $\mathbf{w}_{i}, i \in \mathbb{N}$, form a complete system of $L^{2}$-orthonormal eigenfunctions of the Stokes operator with homogeneous Dirichlet boundary conditions. Similarly, $\psi_{i}, \chi_{i}, i \in \mathbb{N}$, are a basis of $L^{2}$ orthonormal eigenfunctions of the Laplace operator with homogeneous Neumann or Dirichlet boundary conditions, respectively 1 It is well known that the spaces $\bigcup_{n \in \mathbb{N}} \mathcal{V}_{n}, \bigcup_{n \in \mathbb{N}} \mathcal{W}_{n}, \bigcup_{n \in \mathbb{N}} \mathcal{U}_{n}$ are dense in $\mathcal{V}, \mathcal{W}, \mathcal{U}$, respectively. Under condition (H0) for the domains $\Omega$ and $\Omega^{*}$, the elements of $\mathcal{V}_{n}, \mathcal{W}_{n}, \mathcal{U}_{n}$ are bounded. The solutions to be computed are approximated by elements of these spaces,

$$
\begin{aligned}
& \mathbf{v}(t, x) \sim \mathbf{v}^{(n)}(t, x):=\sum_{j=1}^{n} \hat{v}_{j}^{(n)}(t) \mathbf{w}_{j}(x), \\
& \rho(t, x) \sim \rho^{(n)}(t, x):=\sum_{j=1}^{n} \hat{\rho}_{j}^{(n)}(t) \psi_{j}(x), \\
& \phi(t, x) \sim \phi^{(n)}(t, x):=\sum_{j=1}^{n} \hat{\phi}_{j}^{(n)}(t) \psi_{j}(x),
\end{aligned}
$$

\footnotetext{
1 Recall that eigenfunctions for the Laplace operator with homogeneous Neumann boundary conditions are a good choice to discretize corresponding boundary value problems with Robin and also with non-homogeneous Neumann boundary conditions. In contrast, for Dirichlet boundary conditions eigenfunctions for the Laplace operator with homogeneous Dirichlet boundary conditions are to be preferred. Hence our choice of the Faedo-Galerkin method.
} 


$$
\begin{aligned}
& \mu(t, x) \sim \mu^{(n)}(t, x):=\sum_{j=1}^{n} \hat{\mu}_{j}^{(n)}(t) \psi_{j}(x), \\
& V(t, x) \sim V^{(n)}(t, x):=\bar{V}(t, x)+\sum_{j=1}^{n} \hat{V}_{j}^{(n)}(t) \chi_{j}(x) .
\end{aligned}
$$

Generally, we denote Galerkin functions and their coefficients by the same letter and we emphasize coefficients by hats. The Galerkin approximation is given by:

Find $\mathbf{v}^{(n)} \in C^{1}\left(I ; \mathcal{V}_{n}\right), \rho^{(n)}, \phi^{(n)}, \mu^{(n)} \in C^{1}\left(I ; \mathcal{W}_{n}\right), V^{(n)} \in C\left(I ; \mathcal{U}_{n}\right)$ such that for every $t \in I$, $\mathbf{w} \in \mathcal{V}_{n}, \psi_{1}, \psi_{2}, \psi_{3} \in \mathcal{W}_{n}, \chi \in \mathcal{U}_{n}$,

$$
\begin{aligned}
& \int_{\Omega}\left[\mathbf{v}_{t}^{(n)} \cdot \mathbf{w}+\left(\mathbf{v}^{(n)} \cdot \nabla\right) \mathbf{v}^{(n)} \cdot \mathbf{w}+\eta\left(\phi^{(n)}\right) \mathbf{T}\left(\mathbf{v}^{(n)}\right): \mathbf{T}(\mathbf{w})-\mu^{(n)} \nabla \phi^{(n)} \cdot \mathbf{w}+\rho^{(n)} \nabla V^{(n)} \cdot \mathbf{w}\right]=0, \\
& \int_{\Omega}\left[\rho_{t}^{(n)} \psi_{1}+\left(-\rho^{(n)} \mathbf{v}^{(n)}+K\left(\phi^{(n)}\right) \nabla\left(V^{(n)}+\lambda \rho^{(n)}\right)\right) \cdot \nabla \psi_{1}\right]=\int_{\Omega} q \psi_{1}, \\
& \int_{\Omega}\left[\phi_{t}^{(n)} \psi_{2}+\mathbf{v}^{(n)} \cdot \nabla \phi^{(n)} \psi_{2}+M\left(\phi^{(n)}\right) \nabla \mu^{(n)} \cdot \nabla \psi_{2}\right]=0, \\
& \int_{\Omega} \mu^{(n)} \psi_{3}=\int_{\Omega}\left(\delta \nabla \phi^{(n)} \cdot \nabla \psi_{3}+\frac{1}{\delta} W^{\prime}\left(\phi^{(n)}\right) \psi_{3}-\frac{1}{2} \varepsilon^{\prime}\left(\phi^{(n)}\right)\left|\nabla V^{(n)}\right|^{2} \psi_{3}\right) \\
& \int_{\Omega^{*}} \bar{\varepsilon}\left[\phi^{(n)}\right] \nabla V^{(n)} \cdot \nabla \chi=\int_{\Omega} \rho^{(n)} \chi .
\end{aligned}
$$

Existence of solution to the discrete problem. It is advantageous to first eliminate the potential $V$ from all the equations. The discrete version of the equation for the potential can be transformed to the following linear equation for the vector $\hat{V}=\left(\hat{V}_{1}, \ldots, \hat{V}_{n}\right)$ of coefficients:

$$
A_{0}\left(\phi^{(n)}\right) \hat{V}^{(n)}=b_{0}\left(\phi^{(n)}, \rho^{(n)}\right),
$$

where $\left(A_{0}\left(\phi^{(n)}\right)\right)_{i j}=\int_{\Omega^{*}} \bar{\varepsilon}\left[\phi^{(n)}\right] \nabla \chi_{j} \cdot \nabla \chi_{i}$ and

$$
b_{0 i}\left(\phi^{(n)}, \rho^{(n)}\right)=\int_{\Omega^{*}}\left(\rho^{(n)} \chi_{i}-\bar{\varepsilon}\left[\phi^{(n)}\right] \nabla \bar{V} \cdot \nabla \chi_{i}\right) .
$$

Note that we extended $\rho^{(n)}$ by zero to $\Omega^{*} \backslash \bar{\Omega}$. The matrix $A_{0}$ is positive definite, therefore

$$
V^{(n)}=A_{0}^{-1}\left(\phi^{(n)}\right) b_{0}\left(\phi^{(n)}, \rho^{(n)}\right)=V^{(n)}\left(\phi^{(n)}, \rho^{(n)}\right) .
$$

The remaining discrete equations take the form

$$
\begin{aligned}
A_{1} \hat{\mathbf{v}}_{t}^{(n)} & =F_{1}\left(\phi^{(n)}, \rho^{(n)}, \mathbf{v}^{(n)}, \mu^{(n)}\right), \\
-A_{2} \hat{\mu}^{(n)}+B_{1} \hat{\phi}_{t}^{(n)} & =F_{2}\left(\phi^{(n)}, \rho^{(n)}\right), \\
A_{2} \hat{\phi}_{t}^{(n)}+A_{3}\left(\phi^{(n)}\right) \hat{\mu}^{(n)} & =F_{3}\left(\phi^{(n)}, \mathbf{v}^{(n)}\right), \\
A_{2} \hat{\rho}_{t}^{(n)} & =F_{4}\left(\phi^{(n)}, \rho^{(n)}, \mathbf{v}^{(n)}\right) .
\end{aligned}
$$


Here, the stiffness and mass matrices are given as

$$
\begin{aligned}
A_{1} & =\left(\int_{\Omega} \mathbf{w}_{j} \cdot \mathbf{w}_{i}\right)_{i, j=1}^{n}, \\
A_{2} & =\left(\int_{\Omega} \psi_{j} \psi_{i}\right)_{i, j=1}^{n}, \quad B_{1}=\left(\int_{\partial \Omega} \alpha \psi_{i} \psi_{j}\right)_{i, j=1}^{n}, \\
A_{3}(\phi) & =\left(\int_{\Omega} M(\phi) \nabla \psi_{j} \cdot \nabla \psi_{i}\right)_{i, j=1}^{n} .
\end{aligned}
$$

The right-hand sides in 3.10 are defined as

$$
\begin{aligned}
& F_{1}(\phi, \rho, \mathbf{v}, \mu):=\left(-\int_{\Omega}\left(\left(\rho \nabla V^{(n)}(\phi, \rho)+(\mathbf{v} \cdot \nabla) \mathbf{v}-\mu \nabla \phi\right) \mathbf{w}_{i}+\eta(\phi) \mathbf{T}(\mathbf{v}): \mathbf{T}\left(\mathbf{w}_{i}\right)\right)\right)_{i=1}^{n}, \\
& F_{2}(\phi, \rho):=\left(-\int_{\Omega}\left(\delta \nabla \phi \cdot \nabla \psi_{i}+\frac{1}{\delta} W^{\prime}(\phi) \psi_{i}-\frac{1}{2} \varepsilon^{\prime}(\phi)\left|\nabla V^{(n)}(\phi, \rho)\right|^{2} \psi_{i}\right)+\int_{\partial \Omega} \gamma_{f s}^{\prime}(\phi) \psi_{i}\right)_{i=1}^{n}, \\
& F_{3}(\phi, \mathbf{v}):=\left(-\int_{\Omega} \mathbf{v} \cdot \nabla \phi \psi_{i}\right)_{i=1}^{n}, \\
& F_{4}(\phi, \rho, \mathbf{v}):=\left(\int_{\Omega}\left(q \psi_{i}+\rho \mathbf{v} \cdot \nabla \psi_{i}-K(\phi) \nabla\left(V^{(n)}(\phi, \rho)+\lambda \rho\right) \cdot \nabla \psi_{i}\right)\right)_{i=1}^{n} .
\end{aligned}
$$

Our goal is to write this system as an autonomous ODE system

$$
\partial_{t}\left(\begin{array}{l}
\hat{\mathbf{v}}^{(n)} \\
\hat{\phi}^{(n)} \\
\hat{\rho}^{(n)}
\end{array}\right)=F\left(\begin{array}{c}
\hat{\mathbf{v}}^{(n)} \\
\hat{\phi}^{(n)} \\
\hat{\rho}^{(n)}
\end{array}\right) .
$$

Taking the invertibility of $A_{1}$ into account, we note that the first equation of 3.10 ) already has the desired form, provided we are able to express $\hat{\mu}$ as a function of $\hat{\mathbf{v}}, \hat{\phi}$, and $\hat{\rho}$ only. This is possible if the matrix

$$
M=\left(\begin{array}{ccc}
-A_{2} & B_{1} & 0 \\
A_{3}(\phi) & A_{2} & 0 \\
0 & 0 & A_{2}
\end{array}\right)
$$

is regular independently of $\phi$. In this case the last three rows of 3.10 can be transformed by the Gauß algorithm to diagonal form, which implies a representation $\hat{\mu}^{(n)}=\hat{\mu}^{(n)}\left(\phi^{(n)}, \rho^{(n)}, \mathbf{v}^{n}\right)$. Assume $a=(\bar{\mu}, \bar{\phi}, \bar{\rho})^{t}$ satisfies $M a=0$. Multiplying $M a$ by $b:=(\bar{\phi}, \bar{\mu}, 0)^{t}$, we obtain

$$
0=b^{t} M a=\bar{\phi}^{t} B_{1} \bar{\phi}+\bar{\mu}^{t} A_{3} \bar{\mu} .
$$

Since $B_{1}, A_{3}$ are symmetric and positive semidefinite, this implies $B_{1} \bar{\phi}=0$ and $A_{3} \bar{\mu}=0$. Observe that $A_{2}$ is positive definite. From the first component of $M a=0$ we infer that $\bar{\mu}$ is zero. Similarly, the second and third rows imply successively that $\bar{\phi}$ and $\bar{\rho}$ are zero too. Hence the kernel of $M$ is trivial. It is therefore possible to rewrite the discrete system in the form of equations (3.11).

Since all the coefficient functions $\phi \mapsto \eta(\phi), \phi \mapsto \varepsilon(\phi), \phi \mapsto \varepsilon^{\prime}(\phi), \phi \mapsto K(\phi), \phi \mapsto M(\phi)$ and $\phi \mapsto \gamma_{f s}(\phi)$ are Lipschitz, the functions $F_{1}, F_{2}, F_{3}, F_{4}$, and the matrices $A_{0}$ and $M$ are uniformly Lipschitz with respect to $\phi, \rho$, and $\mathbf{v}$, and uniformly bounded if $(\phi, \rho, \mathbf{v})$ is taken from a fixed, bounded subset of $\mathbb{R}^{3 n}$. The inverse of $A_{0}$ is also bounded and Lipschitz, because $\phi \mapsto A_{0}(\phi)$ is Lipschitz, bounded and positive definite with Lipschitz constant and upper and lower bound 
independent of $\phi$. This also implies that the function $(\phi, \rho) \mapsto V^{(n)}(\phi, \rho)$ is Lipschitz and bounded. It remains to verify that the inverse of $M$ is also Lipschitz and bounded. Assume that $M^{-1}(\phi)$ were unbounded for $\phi$ in some compact set. Then there would be a sequence $\left(\phi^{(n)}\right)$ with limit $\phi$ such that the matrix norm of $M^{-1}\left(\phi^{(n)}\right)$ tends to $+\infty$ as $n \rightarrow+\infty$. However, since $M$ is continuous, this implies that $M(\phi)$ has a nontrivial kernel. Hence, for every bounded set $K \subset \mathbb{R}^{n}$ there is a constant $c_{K}$ such that $\left\|M^{-1}(\phi)\right\| \leqslant c_{K}$ for $\phi \in K$. As a consequence, the function $(\mathbf{v}, \phi, \rho) \mapsto F(\mathbf{v}, \phi, \rho)$ in (3.11) is Lipschitz in every bounded set. Hence, applying the Picard-Lindelöf theorem, we obtain a solution of the discrete system that is unique and exists as long as it stays bounded.

Convergence of the discrete solutions. Under the assumptions (H0)-(H5), including (H4-bis), we derive an a priori estimate in the spirit of (3.7) for the discrete system (3.9). The time derivative of (3.9e) for time-independent $\chi$ leads to

$$
\int_{\Omega^{*}} \frac{\mathrm{d}}{\mathrm{d} t}\left(\bar{\varepsilon}\left[\phi^{(n)}\right] \nabla V^{(n)}\right) \cdot \nabla \chi=\int_{\Omega^{*}} \rho_{t}^{(n)} \chi .
$$

For a test function $\chi=V^{(n)}-\bar{V}$ we obtain

$$
\begin{aligned}
& \int_{\Omega^{*}}\left[\frac{\mathrm{d}}{\mathrm{d} t}\left(\frac{1}{2} \bar{\varepsilon}\left[\phi^{(n)}\right]\left|\nabla V^{(n)}\right|^{2}\right)+\frac{1}{2} \varepsilon^{\prime}\left(\phi^{(n)}\right)\right.\left.\phi_{t}^{(n)}\left|\nabla V^{(n)}\right|^{2}-\rho_{t}^{(n)} V^{(n)}\right] \\
&=\int_{\Omega^{*}}\left[\frac{\mathrm{d}}{\mathrm{d} t}\left(\bar{\varepsilon}\left[\phi^{(n)}\right] \nabla V^{(n)}\right) \cdot \nabla \bar{V}-\rho_{t}^{(n)} \bar{V}\right] .
\end{aligned}
$$

Setting $\mathbf{w}=\mathbf{v}^{(n)}$ in (3.9a), $\psi_{1}=V^{(n)}+\lambda \rho^{(n)}$ in (3.9b), $\psi_{2}=\mu^{(n)}$ in (3.9c), $\psi_{3}=\phi_{t}^{(n)}$ in (3.9d) and adding the results to 3.12 yields

$$
\begin{array}{r}
\frac{\mathrm{d}}{\mathrm{d} t}\left[\int_{\Omega}\left\{\frac{1}{2}\left|\mathbf{v}^{(n)}\right|^{2}+\frac{\lambda}{2}\left|\rho^{(n)}\right|^{2}+\frac{\delta}{2}\left|\nabla \phi^{(n)}\right|^{2}+\frac{1}{\delta} W\left(\phi^{(n)}\right)\right\}+\int_{\partial \Omega} \gamma_{f s}\left(\phi^{(n)}\right)+\int_{\Omega^{*}} \frac{1}{2} \bar{\varepsilon}\left[\phi^{(n)}\right]\left|\nabla V^{(n)}\right|^{2}\right] \\
+\int_{\Omega}\left[\eta\left(\phi^{(n)}\right)\left|\mathbf{T}\left(\mathbf{v}^{(n)}\right)\right|^{2}+K\left(\phi^{(n)}\right)\left|\nabla\left(V^{(n)}+\lambda \rho^{(n)}\right)\right|^{2}+M\left(\phi^{(n)}\right)\left|\nabla \mu^{(n)}\right|^{2}\right]+\int_{\partial \Omega} \alpha\left|\phi_{t}^{(n)}\right|^{2} \\
=\int_{\Omega} q\left(V^{(n)}+\lambda \rho^{(n)}\right)+\int_{\Omega^{*}}\left[\frac{\mathrm{d}}{\mathrm{d} t}\left(\bar{\varepsilon}\left[\phi^{(n)}\right] \nabla V^{(n)}\right) \cdot \nabla \bar{V}-\rho_{t}^{(n)} \bar{V}\right] .
\end{array}
$$

Integration with respect to $t$ over $\left(0, t_{0}\right)$ with some $t_{0}$ in combination with the partial integration

$$
\begin{aligned}
\int_{\Omega_{t_{0}}^{*}}\left[\frac{\mathrm{d}}{\mathrm{d} t}\left(\bar{\varepsilon}\left[\phi^{(n)}\right] \nabla V^{(n)}\right) \cdot \nabla \bar{V}-\rho_{t}^{(n)} \bar{V}\right] \\
\quad=\left[\int_{\Omega^{*}}\left(\bar{\varepsilon}\left[\phi^{(n)}\right] \nabla V^{(n)} \cdot \nabla \bar{V}-\rho^{(n)} \bar{V}\right)\right]_{0}^{t_{0}}-\int_{\Omega_{t_{0}^{*}}^{*}}\left[\left(\bar{\varepsilon}\left[\phi^{(n)}\right] \nabla V^{(n)}\right) \cdot \nabla \bar{V}_{t}-\rho^{(n)} \bar{V}_{t}\right]
\end{aligned}
$$

and some Young and Hölder estimates yields the estimate

$$
\begin{array}{r}
\left\|\rho^{(n)}\right\|_{L^{\infty}\left(I ; L^{2}(\Omega)\right)}+\left\|\phi^{(n)}\right\|_{L^{\infty}\left(I ; H^{1}(\Omega)\right)}+\left\|V^{(n)}\right\|_{L^{\infty}\left(I ; H^{1}\left(\Omega^{*}\right)\right)}+\left\|\mathbf{v}^{(n)}\right\|_{L^{\infty}\left(I ; L^{2}(\Omega)\right)} \\
+\left\|\mathbf{v}^{(n)}\right\|_{L^{2}\left(I ; H^{1}(\Omega)\right)}+\left\|\nabla \mu^{(n)}\right\|_{L^{2}\left(\Omega_{T}\right)}+\left\|\left(K\left(\phi^{(n)}\right)\right)^{1 / 2} \nabla \rho^{(n)}\right\|_{L^{2}\left(\Omega_{T}\right)} \\
+\left\|\phi_{t}^{(n)}\right\|_{L^{2}(\partial \Omega \times I)} \leqslant C .
\end{array}
$$

The $L^{\infty}$ bounds for $\phi^{(n)}, \rho^{(n)}$, and $\mathbf{v}^{(n)}$ imply that the solutions to 3.11) exist globally in time. 
Let us prove regularity with respect to time for the velocity field. We denote by $\mathcal{P}_{n}$ the orthogonal $L^{2}$-projection onto $\mathcal{V}_{n}$. We have

LEMMA 3.2 For the approximate solutions to the non-degenerate problem, there is a positive constant $C$ depending on $K_{-}$such that in space dimensions $d \leqslant 3$ and uniformly in $n \in \mathbb{N}$ we have

$$
\left\|\mathbf{v}_{t}^{(n)}\right\|_{L^{6 / 5}\left(I ; \mathcal{V}^{\prime}\right)} \leqslant C .
$$

Proof. We choose $\mathbf{w} \in L^{6}(I ; \mathcal{V})$ arbitrarily and take $\mathcal{P}_{n} \mathbf{w}$ as a test function in the discrete version of the v-equation. Hence, suppressing the index $n$, we have pointwise almost everywhere in time

$$
\begin{aligned}
& \left|\left\langle\mathbf{v}_{t}, \mathbf{w}\right\rangle\right|=\left|\left\langle\mathbf{v}_{t}, \mathcal{P}_{n} \mathbf{w}\right\rangle\right| \\
& \leqslant\left|\int_{\Omega}(\mathbf{v} \cdot \nabla) \mathbf{v} \mathcal{P}_{n} \mathbf{w}\right|+\left|\int_{\Omega} \eta(\phi) \mathbf{T}(\mathbf{v}): \mathbf{T}\left(\mathcal{P}_{n} \mathbf{w}\right)\right|+\left|\int_{\Omega} \mu \nabla \phi \cdot \mathcal{P}_{n} \mathbf{w}\right|+\left|\int_{\Omega} \rho \nabla V \cdot \mathcal{P}_{n} \mathbf{w}\right| \\
& \leqslant C\left\{\|\mathbf{v}\|_{L^{3}(\Omega)}\|\nabla \mathbf{v}\|_{L^{2}(\Omega)}+\|\mathbf{v}\|_{H^{1}(\Omega)}+\|\mu\|_{L^{3}(\Omega)}\|\nabla \phi\|_{L^{2}(\Omega)}+\|\rho\|_{L^{3}(\Omega)}\|\nabla V\|_{L^{2}(\Omega)}\right\}\|\mathbf{w}\|_{H^{1}(\Omega)} .
\end{aligned}
$$

Here, we have used Sobolev's embedding theorem as well as the fact that the projections $\mathcal{P}_{n}$ are uniformly bounded as operators in $\mathcal{L}(\mathcal{V}, \mathcal{V})$. Combining the embedding (cf. [12])

$$
L^{\infty}\left(I ; L^{2}(\Omega)\right) \cap L^{2}\left(I ; H^{1}(\Omega)\right) \hookrightarrow L^{p}\left(\Omega_{T}\right) \quad \text { for } p=\frac{2 d+4}{d}
$$

with the energy estimate 3.13 , we obtain

$$
\begin{aligned}
\left|\int_{0}^{T}\left\langle\mathbf{v}_{t}, \mathbf{w}\right\rangle\right| \leqslant & \|\mathbf{v}\|_{L^{3}\left(\Omega_{T}\right)}\|\nabla \mathbf{v}\|_{L^{2}\left(\Omega_{T}\right)}\|\mathbf{w}\|_{L^{6}\left(I ; H^{1}(\Omega)\right)} \\
& +\|\mu\|_{L^{2}\left(I ; L^{3}(\Omega)\right)}\|\nabla \phi\|_{L^{\infty}\left(I ; L^{2}(\Omega)\right)}\|\mathbf{w}\|_{L^{2}\left(I ; H^{1}(\Omega)\right)} \\
& +\|\mathbf{v}\|_{L^{2}\left(I ; H^{1}(\Omega)\right)}\|\mathbf{w}\|_{L^{2}\left(I ; H^{1}(\Omega)\right)}+\|\rho\|_{L^{3}\left(\Omega_{T}\right)}\|\nabla V\|_{L^{\infty}\left(I ; L^{2}(\Omega)\right)}\|\mathbf{w}\|_{L^{3 / 2}\left(I ; H^{1}(\Omega)\right)} \\
\leqslant & C(T)\|\mathbf{w}\|_{L^{6}\left(I ; H^{1}(\Omega)\right)} .
\end{aligned}
$$

Hence, $\mathbf{v}_{t}^{(n)}$ is uniformly bounded in $L^{6 / 5}\left(I ; \mathcal{V}^{\prime}\right)$.

In a similar fashion, we may prove

COROLlary 3.3 There is a positive constant $C$ independent of $n$ and $K(\cdot)$ such that

$$
\left\|\phi_{t}^{(n)}\right\|_{L^{2}\left(I ; H^{1}(\Omega)^{\prime}\right)}+\sqrt{K_{-}}\left\|\rho_{t}^{(n)}\right\|_{L^{2}\left(I ; H^{1}(\Omega)^{\prime}\right)} \leqslant C .
$$

Together with the compactness results in space (see (3.13), note in particular the uniform positivity of $K(\cdot)$ ) and by use of the Aubin-Lions lemma, we deduce the existence of a subsequence again labeled by $n$ such that

$$
\begin{array}{ll}
\mathbf{v}^{(n)} \rightarrow \mathbf{v} & \text { in } L^{2}\left(I ; H^{1}(\Omega)^{d}\right) \cap W^{1,6 / 5}\left(I ; \mathcal{V}^{\prime}\right) \cap L^{\infty}\left(I ; L^{2}(\Omega)^{d}\right) \\
& \text { and strongly in } L^{2}\left(\Omega_{T}\right), \\
\rho^{(n)} \rightarrow \rho & \text { in } H^{1}\left(I ; H^{1}(\Omega)^{\prime}\right) \cap L^{2}\left(I ; H^{1}(\Omega)\right) \cap L^{\infty}\left(I ; L^{2}(\Omega)\right) \\
& \text { and strongly in } L^{2}\left(\Omega_{T}\right), \\
\phi^{(n)} \rightarrow \phi & \text { in } H^{1}\left(I ; H^{1}(\Omega)^{\prime}\right) \cap L^{\infty}\left(I ; H^{1}(\Omega)\right) \text { and strongly in } L^{2}\left(\Omega_{T}\right), \\
\phi^{(n)} \rightarrow \phi & \text { strongly in } L^{2}(\partial \Omega \times I),
\end{array}
$$




$$
\begin{aligned}
\phi_{t}^{(n)} & \rightarrow \phi_{t} & & \text { in } L^{2}(\partial \Omega \times I), \\
\phi^{(n)}(t, x) & \rightarrow \phi(t, x) & & \text { for almost every } t \in I, x \in \Omega, \\
\nabla \mu^{(n)} & \rightarrow \nabla \mu & & \text { in } L^{2}\left(\Omega_{T}\right), \\
V^{(n)} & { }^{*} V & & \text { in } L^{\infty}\left(I ; H^{1}\left(\Omega^{*}\right)\right), \\
\frac{1}{2} \varepsilon^{\prime}\left(\phi^{(n)}\right)\left|\nabla V^{(n)}\right|^{2} & \rightarrow \Theta & & \text { in the sense of measures. }
\end{aligned}
$$

By compensated compactness (see [11], [35]), we obtain easily

$$
\mathbf{v}^{(n)} \cdot \nabla \rho^{(n)} \rightarrow \mathbf{v} \cdot \nabla \rho \quad \text { and } \quad \mathbf{v}^{(n)} \cdot \nabla \phi^{(n)} \rightarrow \mathbf{v} \cdot \nabla \phi \quad \text { in } L^{p}\left(\Omega_{T}\right),
$$

for some $p>1$, since $\nabla \cdot \mathbf{v}^{(n)}=0$ and $\nabla \rho^{(n)}, \nabla \phi^{(n)}$ are $L^{2}$-integrable gradient fields. Since $\phi^{(n)} \rightarrow \phi$ almost everywhere, we also have for a subsequence

$$
\varepsilon\left(\phi^{(n)}\right) \rightarrow \varepsilon(\phi), \quad \varepsilon^{\prime}\left(\phi^{(n)}\right) \rightarrow \varepsilon^{\prime}(\phi) \quad \text { and } \quad \eta\left(\phi^{(n)}\right) \rightarrow \eta(\phi)
$$

almost everywhere. We recall the boundedness of $\varepsilon, \varepsilon^{\prime}$, and $\eta$ and obtain easily the strong convergence in $L^{p}\left(\Omega_{T}\right)$ for arbitrary $p<\infty$. These convergences allow us to pass to the limit in the Galerkin discretization. In equation (3.5a) the convergence

$$
\int_{\Omega_{T}} \rho^{(n)} \nabla V^{(n)} \cdot \mathbf{w} \rightarrow \int_{\Omega_{T}} \rho \nabla V \cdot \mathbf{w}
$$

follows from the strong convergence of $\rho$ in $L^{2}\left(\Omega_{T}\right)$; the convergence

$$
\int_{\Omega_{T}} \mu^{(n)} \nabla \phi^{(n)} \cdot \mathbf{w} \rightarrow \int_{\Omega_{T}} \mu \nabla \phi \cdot \mathbf{w}
$$

is derived after an integration by parts from

$$
\int_{\Omega_{T}} \phi^{(n)} \nabla \mu^{(n)} \cdot \mathbf{w} \rightarrow \int_{\Omega_{T}} \phi \nabla \mu \cdot \mathbf{w} .
$$

As a consequence, the limit functions $\mathbf{v}, \rho, \phi, \mu, V$ solve the system (3.5) except for (3.5d) which has to be replaced by

$$
\begin{aligned}
\int_{\Omega_{T}} \mu \psi_{3}= & \int_{\Omega_{T}}\left(\delta \nabla \phi \cdot \nabla \psi_{3}+\frac{1}{\delta} W^{\prime}(\phi) \psi_{3}\right) \\
& -\int_{0}^{T}\left\langle\Theta, \psi_{3}\right\rangle_{\mathcal{M}(\Omega) \times L^{\infty}(\Omega)}+\int_{0}^{T} \int_{\partial \Omega}\left(\alpha \phi_{t}+\gamma_{f S}^{\prime}(\phi)\right) \psi_{3} .
\end{aligned}
$$

It remains to prove $\Theta=\frac{1}{2} \varepsilon^{\prime}(\phi)|\nabla V|^{2}$. It is sufficient to show that the convergence $V^{(n)} \rightarrow V$ is strong in $L^{2}\left(I ; H^{1}(\Omega)\right)$ as it implies

$$
\begin{aligned}
& \left\|\varepsilon^{\prime}\left(\phi^{(n)}\right)\left|\nabla V^{(n)}\right|^{2}-\varepsilon^{\prime}(\phi)|\nabla V|^{2}\right\|_{L^{1}\left(\Omega_{T}\right)} \\
& \leqslant\left\|\varepsilon^{\prime}\left(\phi^{(n)}\right)\left(\left|\nabla V^{(n)}\right|^{2}-|\nabla V|^{2}\right)\right\|_{L^{1}\left(\Omega_{T}\right)}+\left\|\left(\varepsilon^{\prime}\left(\phi^{(n)}\right)-\varepsilon^{\prime}(\phi)\right)|\nabla V|^{2}\right\|_{L^{1}\left(\Omega_{T}\right)} \\
& \leqslant\left\|\varepsilon^{\prime}\left(\phi^{(n)}\right)\right\|_{L^{\infty}\left(\Omega_{T}\right)}\left\|\nabla\left(V^{(n)}-V\right)\right\|_{L^{2}\left(\Omega_{T}\right)}\left\|\nabla\left(V^{(n)}+V\right)\right\|_{L^{2}\left(\Omega_{T}\right)} \\
& \quad+\left\|\left(\varepsilon^{\prime}\left(\phi^{(n)}\right)-\varepsilon^{\prime}(\phi)\right)|\nabla V|^{2}\right\|_{L^{1}\left(\Omega_{T}\right)} \\
& \rightarrow 0 \quad \text { as } n \rightarrow \infty .
\end{aligned}
$$

The last term here converges to zero due to the Lebesgue dominated convergence theorem. 
In order to prove the strong convergence of $V^{(n)}$ in $L^{2}\left(I ; H^{1}(\Omega)\right)$ we take the test function $V^{(n)}-\bar{V}-P_{n}(V-\bar{V})$ in the Galerkin discretization $(3.9 \mathrm{e})$ with solution $V^{(n)}$ and $V-V^{(n)}$ in (3.5e) with solution $V$, add both relations and integrate over $t$. Here $P_{n}$ is the orthogonal projection of $H_{0}^{1}\left(\Omega^{*}\right)$ onto $\mathcal{U}_{n}$. This projection has $\left\|P_{n}\right\|=1$. Since $\left\|\nabla\left(P_{n}(V-\bar{V})-(V-\bar{V})\right)\right\|_{L^{2}\left(\Omega^{*}\right)}$ tends to 0 as $n \rightarrow \infty$ for almost every $t \in I$, we obtain by the Lebesgue dominated convergence theorem

$$
\lim _{n \rightarrow \infty} \| \nabla\left(P_{n}(V-\bar{V})-(V-\bar{V}) \|_{L^{2}\left(\Omega_{T}^{*}\right)}=0 .\right.
$$

We have

$$
\begin{aligned}
\int_{I} \int_{\Omega^{*}} \bar{\varepsilon}\left[\phi^{(n)}\right]\left|\nabla\left(V^{(n)}-V\right)\right|^{2} \leqslant & \int_{I} \int_{\Omega^{*}} \bar{\varepsilon}\left[\phi^{(n)}\right] \nabla V^{(n)} \cdot \nabla\left(P_{n}(V-\bar{V})-(V-\bar{V})\right) \\
& +\int_{\Omega_{T}}\left(\varepsilon\left(\phi^{(n)}\right)-\varepsilon(\phi)\right) \nabla V \cdot \nabla\left(V-V^{(n)}\right)+R^{(n)}
\end{aligned}
$$

where

$$
R^{(n)}:=\left|\int_{\Omega_{T}}\left(\rho^{(n)}-\rho\right)\left(V^{(n)}-V\right)+\int_{\Omega_{T}} \rho^{(n)}\left(V-\bar{V}-P_{n}(V-\bar{V})\right)\right| .
$$

Observe that $R^{(n)}=o_{n}(1)$. Indeed, using (H0), the compact embedding $L^{2}(\Omega) \hookrightarrow \hookrightarrow H^{1}(\Omega)^{\prime}$ as well as the continuous embedding $H^{1}(\Omega)^{\prime} \hookrightarrow W^{1, p}(\Omega)^{\prime}$ for $p>2$, we infer from 3.8, 3.13 and the Aubin-Lions lemma the existence of a subsequence again labeled by $n$ such that $\rho_{n}$ strongly converges to $\rho$ in $L^{2}\left(I ; H^{1}(\Omega)^{\prime}\right)$. Again by 3.13 , (a subsequence of) $V^{(n)}-V$ converges to zero in the weak topology of $L^{2}\left(I ; H^{1}(\Omega)\right)$. Together with 3.17), this shows the $o_{n}(1)$-property of $R^{(n)}$. Altogether,

$$
\begin{aligned}
\left\|\nabla\left(V^{(n)}-V\right)\right\|_{L^{2}\left(\Omega_{T}^{*}\right)}^{2} \leqslant & C_{1}\left\|\nabla V^{(n)}\right\|_{L^{2}\left(\Omega_{T}^{*}\right)}\left\|\nabla\left(P_{n}(V-\bar{V})-(V-\bar{V})\right)\right\|_{L^{2}\left(\Omega_{T}^{*}\right)} \\
& +C_{2}\left\|\left(\varepsilon\left(\phi^{(n)}\right)-\varepsilon(\phi)\right) \nabla V\right\|_{L^{2}\left(\Omega_{T}\right)}^{2}+o_{n}(1) \\
\rightarrow & 0 .
\end{aligned}
$$

The second norm here again converges due to the Lebesgue dominated convergence theorem.

We have thus proved the following existence theorem:

TheOREM 3.4 Let $\Omega, \Omega^{*}$ satisfy assumption (H0) and suppose $\varepsilon(\cdot), \eta(\cdot), M(\cdot), \gamma_{f s}(\cdot), K(\cdot)$, and $\bar{V}$ satisfy assumptions (H1)-(H4-bis) and (H5). Assume the initial data $\rho_{0}, \phi_{0}, \mathbf{v}_{0}$ are in $L^{2}(\Omega), H^{1}(\Omega ;[-1,1])$, and $\mathcal{V}$, respectively. Then there is a quintuple $(\rho, \phi, \mathbf{v}, \mu, V)$ with $\rho \in$ $L^{\infty}\left(I ; L^{2}(\Omega)\right) \cap L^{2}\left(I ; H^{1}(\Omega)\right) \cap H^{1}\left(I ; H^{1}(\Omega)^{\prime}\right), \phi \in L^{\infty}\left(I ; H^{1}(\Omega)\right) \cap H^{1}\left(I ; H^{1}(\Omega)^{\prime}\right) \cap$ $H^{1}\left(I ; L^{2}(\partial \Omega)\right), \mathbf{v} \in L^{\infty}\left(I ; L^{2}(\Omega)\right) \cap L^{2}\left(I ; H^{1}(\Omega)\right) \cap W^{1,6 / 5}\left(I ; \mathcal{V}^{\prime}\right), \mu \in L^{2}\left(I ; H^{1}(\Omega)\right)$, and $V \in L^{\infty}\left(I ; H^{1}(\Omega)\right)$ which solves 3.1)-3.4 in the sense of Definition 3.1

\subsection{The case of degenerate conductivity-higher regularity for the phase-field}

We now consider the case where the electric conductivity $K(\phi)$ is zero for $\phi \leqslant-1$. In this case the charge transport equation is degenerate parabolic, and we cannot expect to obtain an estimate for $\nabla \rho$ in $L^{2}\left(\Omega_{T}\right)$. As a consequence, time-compactness for the velocity field has to be established differently. For the existence proof, we proceed as follows. We first approximate the problem by a family of problems with non-degenerate conductivities $\left(K_{\eta}(\phi)\right)_{\eta \rightarrow 0}$ satisfying (H4) and (H4-bis) 
such that $\left|K_{\eta}(\phi)-K(\phi)\right| \leqslant \eta$. Theorem 3.4 gives the existence of solutions $\left(\mathbf{v}_{\eta}, \rho_{\eta}, \phi_{\eta}, V_{\eta}, \mu_{\eta}\right)$ to this problem.

We plan to pass to the limit $\eta \rightarrow 0$ for a suitable weakly convergent subsequence. It will be crucial to prove some more regularity for the phase-field $\phi$ in the interior of $\Omega$ to compensate for the weak regularity of $\rho$ when passing to the limit in nonlinear terms like $K(\phi) \nabla \rho \cdot \mathbf{w}$. Moreover, we have to prove $W^{1,6 / 5}\left(I ; \mathcal{V}^{\prime}\right)$-regularity for the flow field $\mathbf{v}_{\eta}$ uniformly in $\eta \rightarrow 0$ without using any integrability estimates on $\nabla \rho$. We begin with a result on higher regularity of the electrostatic potential.

Lemma 3.5 Let $\bar{V}$ satisfy (H5). For $\eta>0$, consider the solution $\left(\mathbf{v}_{\eta}, \rho_{\eta}, \phi_{\eta}, V_{\eta}, \mu_{\eta}\right)$ of the nondegenerate problem (3.5) corresponding to the non-degenerate conductivity $K_{\eta}$. Then there exists an exponent $p>1$ and a positive constant $C$ independent of $\eta$ such that

$$
\left\|\nabla V_{\eta}\right\|_{L^{\infty}\left(I ; L^{2 p}\left(\Omega^{*}\right)\right)} \leqslant C .
$$

Proof. For ease of presentation we omit the index $\eta$ throughout this proof. To guarantee homogeneous Dirichlet boundary conditions, we write the last equation of system 3.1 in the form

$$
-\nabla \cdot(\bar{\varepsilon}[\phi] \nabla(V-\bar{V}))=\bar{\rho}+\nabla \cdot(\bar{\varepsilon}[\phi] \nabla \bar{V})
$$

where $\bar{\rho}$ is defined as

$$
\bar{\rho}:= \begin{cases}\rho & \text { in }(0, T] \times \bar{\Omega}, \\ 0 & \text { in }(0, T] \times\left(\bar{\Omega}^{*} \backslash \bar{\Omega}\right) .\end{cases}
$$

Obviously, $\bar{\rho} \in L^{\infty}\left(I ; L^{2}\left(\Omega^{*}\right)\right)$. Similarly, $\bar{\varepsilon}\left[\phi^{(n)}\right] \nabla \bar{V} \in L^{\infty}\left(I ; L^{r}\left(\Omega^{*}\right)\right)$ by assumption (H5). Observe that $\varepsilon(\cdot)$ is uniformly bounded from above and from below independently of $\eta$. Hence, we may apply a standard result on $p$-integrability of gradients (cf. Theorem 2.1 in [4]) to conclude.

This result is the key to establishing locally higher regularity in the space for the phase-field. We have

Lemma 3.6 Let $\Omega^{\prime} \subset \subset \Omega$ and $d=2$, 3. If $d=3$, assume in addition $\varepsilon(\cdot) \equiv$ const. Then there exist

$$
p^{*}> \begin{cases}1 & \text { if } d=2, \\ 6 / 5 & \text { if } d=3,\end{cases}
$$

and a positive constant $C_{p^{*}}$ which does not depend on $\eta$ such that

$$
\left\|\phi_{\eta}\right\|_{L^{2}\left(I ; W^{2, p^{*}}\left(\Omega^{\prime}\right)\right)}<C_{p^{*}} .
$$

REMARK 3.7 Note that $W^{2, p^{*}}\left(\Omega^{\prime}\right) \hookrightarrow \hookrightarrow H^{1}\left(\Omega^{\prime}\right)$ in space dimensions $d=2,3$.

Proof. By the $W^{2, p}$-interior estimates for Poisson's equation (see e.g. [16, Theorem 9.11]), applied to

$$
-\Delta \phi_{\eta}=\delta^{-1} \mu_{\eta}-\delta^{-2} W^{\prime}\left(\phi_{\eta}\right)+\frac{1}{2 \delta} \varepsilon^{\prime}\left(\phi_{\eta}\right)\left|\nabla V_{\eta}\right|^{2}=: f_{\eta},
$$

we obtain for $f_{\eta} \in L^{p}(\Omega)$

$$
\left\|\phi_{\eta}\right\|_{W^{2, p}\left(\Omega^{\prime}\right)} \leqslant C\left(\left\|f_{\eta}\right\|_{L^{p}(\Omega)}+\left\|\phi_{\eta}\right\|_{L^{2}(\Omega)}\right)
$$


with $C$ depending only on $\Omega$ and $\Omega^{\prime}$. The main task therefore is to prove that $f_{\eta}$ is bounded in $L^{p}\left(\Omega_{T}\right)$ uniformly with respect to $\eta$. From $(3.7)$ and the embedding $H^{1}(\Omega) \subset L^{6}(\Omega)$ (which holds for space dimensions $d \leqslant 3$ ), we conclude that $W^{\prime}\left(\phi_{\eta}\right)$ and $\mu_{\eta}$ are bounded in $L^{2}\left(\Omega_{T}\right)$. Hence the main difficulty is to handle the term $\frac{1}{2 \delta} \varepsilon^{\prime}\left(\phi_{\eta}\right)\left|\nabla V_{\eta}\right|^{2}$ as the a priori estimate (3.7) reduces to an estimate of this term in $L^{\infty}\left(I ; L^{1}(\Omega)\right)$ only. If $\varepsilon(\phi)$ is independent of $\phi$, then this term disappears, and 3.21 is proved with $p=2$. This gives the result for $d=3$.

We now concentrate on the case $d=2, \varepsilon(\cdot)$ depending on the phase-field $\phi$. Lemma 3.5 provides a uniform bound for $\|\nabla V\|_{L^{\infty}\left(I ; L^{2 p}(\Omega)\right)}$ with $p>1$. As a result, the term $\varepsilon^{\prime}\left(\phi_{\eta}\right)\left|\nabla V_{\eta}\right|^{2}$ is bounded in $L^{\infty}\left(I ; L^{p}(\Omega)\right)$ uniformly with respect to $\eta$, with some $p>1$ independent of $\eta$. This gives the result for $d=2$.

Next, we prove

LEMmA 3.8 Let $d \in\{2,3\}$. Then there exists a constant $C>0$ independent of $\eta>0$ such that

$$
\left\|\partial_{t} \mathbf{v}_{\eta}\right\|_{L^{6 / 5}\left(I ; \mathcal{S}_{d}^{\prime}\right)} \leqslant C \text {. }
$$

Proof. The proof mimics the one of Lemma 3.2. The only term to be treated differently is $\int_{\Omega_{T}} \rho_{\eta} \nabla V_{\eta} \cdot \mathbf{w}$. Using the $L^{\infty}\left(I ; L^{2 p}\left(\Omega^{*}\right)\right)$-regularity established in Lemma 3.5 for $\nabla V_{\eta}$ with some $p>1$ and also Sobolev's embedding result, we may estimate, with a parameter $q$ satisfying $1 / 2+1 /(2 p)+1 / q=1$

$$
\begin{aligned}
\left|\int_{\Omega_{T}} \rho_{\eta} \nabla V_{\eta} \cdot \mathbf{w}\right| & \leqslant C \int_{0}^{T}\left\|\rho_{\eta}\right\|_{L^{2}(\Omega)}\left\|\nabla V_{\eta}\right\|_{L^{2 p}(\Omega)}\|\mathbf{w}\|_{L^{q}(\Omega)} \\
& \leqslant C\left\|\rho_{\eta}\right\|_{L^{\infty}\left(I ; L^{2}(\Omega)\right)}\left\|\nabla V_{\eta}\right\|_{L^{\infty}\left(I ; L^{2 p}(\Omega)\right)}\|\mathbf{w}\|_{L^{2}\left(I ; W^{1, d}(\Omega)\right)} .
\end{aligned}
$$

Using the fact that $\mathcal{S}_{d}$ is a closed subspace of $\left(W_{0}^{1, d}(\Omega)\right)^{d}, d \in\{2,3\}$, and therefore reflexive, we may combine the Aubin-Lions lemma with the uniform bounds on $\left\|\mathbf{v}_{\eta}\right\|_{L^{2}(I ; \mathcal{V})}$ and on $\left\|\partial_{t} \mathbf{v}_{\eta}\right\|_{W^{1,6 / 5}\left(I ; \mathcal{S}_{d}^{\prime}\right)}$ to establish

COROllary 3.9 For $\eta \rightarrow 0$, there is a subsequence $\eta^{\prime} \rightarrow 0$ such that $\left(\mathbf{v}_{\eta^{\prime}}\right)_{\eta^{\prime} \rightarrow 0}$ converges strongly in $L^{2}\left(\Omega_{T}\right)$ to a flow field $\mathbf{v} \in L^{2}\left(\Omega_{T}\right)$.

Summing up, we infer the existence of a subsequence of solutions, again denoted by $\left(\mathbf{v}_{\eta}, \rho_{\eta}, \phi_{\eta}, V_{\eta}, \mu_{\eta}\right)$, such that the following convergences hold:

$$
\begin{aligned}
\mathbf{v}_{\eta} \rightarrow \mathbf{v} & \text { in } L^{2}(I ; \mathcal{V}) \cap W^{1,6 / 5}\left(I ; \mathcal{S}_{d}^{\prime}\right) \text { and strongly in } L^{2}\left(\Omega_{T}\right), \\
\rho_{\eta} \rightarrow \rho & \text { in } L^{\infty}\left(I ; L^{2}(\Omega)\right), \\
\phi_{\eta} \rightarrow \phi & \text { in } H^{1}\left(I ; H^{1}(\Omega)^{\prime}\right) \cap L^{\infty}\left(I ; H^{1}(\Omega)\right) \cap H^{1}\left(I ; L^{2}(\partial \Omega)\right) \\
& \text { and strongly in } L^{2}\left(\Omega_{T}\right), \\
\mu_{\eta} \rightarrow \mu & \text { in } L^{2}\left(I ; H^{1}(\Omega)\right), \\
V_{\eta} \rightarrow V & \text { in } L^{\infty}\left(I ; W^{1,2 p}\left(\Omega^{*}\right)\right) \quad \text { for some } p>1, \\
\frac{1}{2} \varepsilon^{\prime}\left(\phi_{\eta}\right)\left|\nabla V_{\eta}\right|^{2} \rightarrow \Theta & \text { in } L^{\infty}\left(I ; L^{p}\left(\Omega^{*}\right)\right) \quad \text { for some } p>1 .
\end{aligned}
$$

By passing to a further subsequence we have

$$
K\left(\phi_{\eta}\right) \nabla \rho_{\eta} \rightarrow \Psi \quad \text { in } L^{2}\left(\Omega_{T}\right) .
$$


We now have to identify $\Psi$ with $K(\phi) \nabla \rho$. Let $\psi \in C_{0}^{\infty}\left(\Omega_{T}\right)$ be a test function and $\Omega^{\prime} \subset \subset \Omega$ be a domain such that supp $\psi \subset I \times \Omega^{\prime}$ and $\operatorname{dist}\left(\Omega^{\prime}, \partial \Omega\right)>0$. Note that the regularity estimate (3.20) combined with the uniform bound for $\phi_{\eta}$ in $H^{1}\left(I ; H^{1}(\Omega)^{\prime}\right)$, the compact imbedding $W^{2, p}\left(\Omega^{\prime}\right) \hookrightarrow$ $H^{1}\left(\Omega^{\prime}\right)$ ( $p$ the exponent in $(3.20)$ ), and the Aubin-Lions lemma gives a subsequence $\phi_{\eta^{\prime}}$ which strongly converges in $L^{2}\left(I ; H^{1}\left(\Omega^{\prime}\right)\right)$ to $\phi$.

Integration by parts yields

$$
\int_{\Omega_{T}} K\left(\phi_{\eta}\right) \nabla \rho_{\eta} \cdot \psi=-\int_{\Omega_{T}} \rho_{\eta}\left(K^{\prime}\left(\phi_{\eta}\right) \nabla \phi_{\eta} \cdot \psi+K\left(\phi_{\eta}\right) \nabla \cdot \psi\right) .
$$

Here the right-hand side converges to

$$
-\int_{\Omega_{T}} \rho\left(K^{\prime}(\phi) \nabla \phi \cdot \psi+K(\phi) \nabla \cdot \psi\right)
$$

while the limit of the left-hand side is $\int_{\Omega_{T}} \Psi \cdot \psi$. This shows $\Psi=K(\phi) \nabla \rho$ in the sense of distributions.

The convergences we have established allow us to pass to the limit $\eta \rightarrow 0$ in the weak formulation of the problem for smooth test functions. The limits in the convective terms are taken after an integration by parts,

$$
\int_{\Omega_{T}} \mathbf{v}_{\eta} \cdot \nabla \rho_{\eta} \psi=-\int_{\Omega_{T}} \rho_{\eta} \mathbf{v}_{\eta} \cdot \nabla \psi \rightarrow-\int_{\Omega_{T}} \rho \mathbf{v} \cdot \nabla \psi
$$

Here we use the weak convergence $\rho_{\eta} \rightarrow \rho$ in $L^{2}\left(\Omega_{T}\right)$ and the strong convergence of $\left(\mathbf{v}_{\eta}\right)_{\eta \rightarrow 0}$ in $L^{2}\left(\Omega_{T}\right)$. The other limits are the same as in the Galerkin approximation in Section 3.3 The strong convergence $V_{\eta} \rightarrow V$ in $L^{2}\left(I ; H^{1}\left(\Omega^{*}\right)\right)$ is also proved as before, giving rise to the identification

$$
\lim _{\eta \rightarrow 0} \varepsilon^{\prime}\left(\phi_{\eta}\right)\left|\nabla V_{\eta}\right|^{2}=\varepsilon^{\prime}(\phi)|\nabla V|^{2} .
$$

Hence the following theorem is verified:

THEOREM 3.10 Let $\Omega, \Omega^{*}$ satisfy assumption (H0) and suppose $\eta(\cdot), \varepsilon(\cdot), M(\cdot), \gamma_{f s}(\cdot), K(\cdot)$, and $\bar{V}$ to be in accordance with (H1)-(H4) and (H5). Assume the initial data $\rho_{0}, \phi_{0}, \mathbf{v}_{0}$ to be given in $L^{2}(\Omega), H^{1}(\Omega ;[-1,1])$, and $\mathcal{V}$, respectively. Let $d=2,3$, and for $d=3$ assume in addition that $\varepsilon$ is independent of $\phi$. Then there is a quintuple $(\rho, \phi, \mathbf{v}, \mu, V)$ which solves $(3.1)-(3.3)$ in the sense of Definition 3.1.

Note that uniqueness remains an open problem due to the intricate coupling of the various terms involved. On account of its importance for control problems, we intend to come back to this issue in the future.

REMARK 3.11 By Lemma 3.5 . $\nabla V$ is an element of $L^{\infty}\left(I ; L^{2 p}\left(\Omega^{*}\right)\right)$ for a parameter $p>1$. In space dimension $d=2$, this is sufficient to show that for almost each $t \in I$ equation (3.5a) defines a linear functional on $\left(H_{0}^{1}(\Omega)\right)^{d}$ which vanishes on $\mathcal{V}$. Therefore, we may apply standard methods (e.g. Lemma 2.1 of [17]) to prove the existence of a pressure field $p(\cdot, t) \in L^{2}(\Omega)$ such that for almost every $t \in I$,

$$
\left\langle\mathbf{v}_{t}, \mathbf{w}\right\rangle_{1}+\int_{\Omega}((\mathbf{v} \cdot \nabla) \mathbf{v} \cdot \mathbf{w}+\eta(\phi) \mathbf{T}(\mathbf{v}): \mathbf{T}(\mathbf{w})-\mu \nabla \phi \cdot \mathbf{w}+\rho \nabla V \cdot \mathbf{w})=-\langle\operatorname{grad} p, \mathbf{w}\rangle_{1}
$$

for all $\mathbf{w} \in\left(H_{0}^{1}(\Omega)\right)^{d}$. 


\section{The case of negligible acceleration terms in the flow field}

As we expect the effect of acceleration terms in the equation for the velocity field to be small in general, it is worthwhile to study also the following system based on the quasi-stationary Stokes approximation:

$$
\begin{array}{ll}
-\nabla \cdot(\eta(\phi) \mathbf{T}(\mathbf{v}))+\nabla p-\mu \nabla \phi+\rho \nabla V=0 & \text { in } \Omega_{T}, \\
\rho_{t}+\mathbf{v} \cdot \nabla \rho-\nabla \cdot(K(\phi) \nabla(V+\lambda \rho))=q & \text { in } \Omega_{T}, \\
\phi_{t}+\mathbf{v} \cdot \nabla \phi-\nabla \cdot(M(\phi) \nabla \mu)=0 & \text { in } \Omega_{T}, \\
\mu=-\delta \Delta \phi+\frac{1}{\delta} W^{\prime}(\phi)-\frac{1}{2} \varepsilon^{\prime}(\phi)|\nabla V|^{2} & \text { in } \Omega \forall t \in(0, T), \\
-\nabla \cdot(\bar{\varepsilon}[\phi] \nabla V)=\rho & \text { in } \Omega^{*} \forall t \in(0, T) .
\end{array}
$$

Keeping the boundary conditions 3.3 , 3.4 and slightly modifying the argument of Subsection 3.2 , the following a priori estimates can be easily derived:

$$
\begin{array}{r}
\|\rho\|_{L^{\infty}\left(I ; L^{2}(\Omega)\right)}+\|\phi\|_{L^{\infty}\left(I ; H^{1}(\Omega)\right)}+\|W(\phi)\|_{L^{\infty}\left(I ; L^{1}(\Omega)\right)}+\|V\|_{L^{\infty}\left(I ; H^{1}\left(\Omega^{*}\right)\right)}+\|\mathbf{v}\|_{L^{2}\left(I ; H^{1}(\Omega)\right)} \\
+\left\|K(\phi)^{1 / 2} \nabla \rho\right\|_{L^{2}\left(\Omega_{T}\right)}+\|\nabla \mu\|_{L^{2}\left(\Omega_{T}\right)}+\left\|\phi_{t}\right\|_{L^{2}(\partial \Omega \times I)} \leqslant C .
\end{array}
$$

Similarly,

$$
\left\|\phi_{t}\right\|_{L^{2}\left(I ; H^{1}(\Omega)^{\prime}\right)}+\left\|\rho_{t}\right\|_{L^{2}\left(I ; W^{1, p}(\Omega)^{\prime}\right)} \leqslant C
$$

with

and arbitrary positive $\varepsilon$.

$$
p= \begin{cases}2+\varepsilon, & d=2, \\ 3, & d=3,\end{cases}
$$

Following similar strategies to those in Section 3, we obtain an existence result.

THEOREM 4.1 Let $\Omega, \Omega^{*}$ satisfy assumption (H0) and suppose $\eta(\cdot), \varepsilon(\cdot), M(\cdot), \gamma_{f s}(\cdot), K(\cdot)$, and $\bar{V}$ to be in accordance with (H1)-(H4) and (H5). Assume the initial data $\rho_{0}, \phi_{0}$ to be given in $L^{2}(\Omega), H^{1}(\Omega ;[-1,1])$. Let $d=2,3$, and for $d=3$ assume in addition that $\varepsilon$ is independent of $\phi$. Then there is a quintuple $(\rho, \phi, \mathbf{v}, \mu, V)$ with $\rho \in L^{\infty}\left(I ; L^{2}(\Omega)\right) \cap H^{1}\left(I ; W^{1, p}(\Omega)^{\prime}\right), \phi \in$ $L^{\infty}\left(I ; H^{1}(\Omega)\right) \cap H^{1}\left(I ; H^{1}(\Omega)^{\prime}\right) \cap H^{1}\left(I ; L^{2}(\partial \Omega)\right), \mathbf{v} \in L^{2}(I ; \mathcal{V}), \mu \in L^{2}\left(I ; H^{1}(\Omega)\right)$, and $V \in$ $L^{\infty}\left(I ; H^{1}(\Omega)\right)$ which solves $4.1,3.3,3.4$ in the following sense:

$$
\begin{aligned}
& \int_{\Omega}[\eta(\phi) \mathbf{T}(\mathbf{v}): \mathbf{T}(\mathbf{w})+(\rho \nabla V-\mu \nabla \phi) \cdot \mathbf{w}]=0 \quad \forall t \in I \\
& \int_{0}^{T}\left\langle\rho_{t}, \psi_{1}\right\rangle_{2}+\int_{\Omega_{T}}(K(\phi) \nabla(V+\lambda \rho)-\rho \mathbf{v}) \cdot \nabla \psi_{1}=\int_{\Omega_{T}} q \psi_{1}, \\
& \int_{0}^{T}\left\langle\phi_{t}, \psi_{2}\right\rangle_{3}+\int_{\Omega_{T}}\left(\mathbf{v} \cdot \nabla \phi \psi_{2}+M(\phi) \nabla \mu \cdot \nabla \psi_{2}\right)=0, \\
& \int_{\Omega_{T}} \mu \psi_{3}=\int_{\Omega_{T}}\left(\delta \nabla \phi \cdot \nabla \psi_{3}+\frac{1}{\delta} W^{\prime}(\phi) \psi_{3}-\frac{1}{2} \varepsilon^{\prime}(\phi)|\nabla V|^{2} \psi_{3}\right)+\int_{0}^{T} \int_{\partial \Omega}\left(\alpha \phi_{t}+\gamma_{f s}^{\prime}(\phi)\right) \psi_{3}, \\
& \int_{\Omega_{T}^{*}} \bar{\varepsilon}[\phi] \nabla V \cdot \nabla \chi=\int_{\Omega_{T}} \rho \chi
\end{aligned}
$$


for every $\mathbf{w} \in \mathcal{V}, \psi_{1} \in W^{1, \infty}\left(\Omega_{T}\right), \psi_{2} \in L^{2}\left(I ; H^{1}(\Omega)\right), \psi_{3} \in L^{2}\left(I ; H^{1}(\Omega)\right) \cap L^{1}\left(I ; L^{\infty}(\Omega)\right)$, and $\chi \in L^{2}(I ; \mathcal{U})$. Here, $\langle\cdot, \cdot\rangle_{2}$ and $\langle\cdot, \cdot\rangle_{3}$ denote the dual pairings on the spaces $L^{2}\left(I ; W^{1, p}(\Omega)\right)$, and $L^{2}\left(I ; H^{1}(\Omega)\right)$, respectively.

REMARK 4.2 Let us indicate the main changes in the proof.

- Instead of (3.10), we write the discrete system with right-hand sides depending only on $\phi$ and $\rho$. One easily observes that the corresponding matrix on the left-hand side is bijective, hence we may express $\bar{\mu}$ and $\overline{\mathbf{v}}$ in terms of $\bar{\phi}$ and $\bar{\rho}$ only. Local existence and uniqueness follows immediately by Picard-Lindelöf.

- The existence proof for the non-degenerate auxiliary problems as well as for the degenerate problem cannot rely on strong convergence of the velocity field any longer. This affects in particular the passage to the limit in the convective term $\mathbf{v}_{\eta} \cdot \nabla \rho_{\eta} \psi$ (cf. equation (3.23)). We proceed as follows:

$$
\int_{\Omega_{T}} \mathbf{v}_{\eta} \cdot \nabla \rho_{\eta} \psi=-\int_{\Omega_{T}} \rho_{\eta} \mathbf{v}_{\eta} \cdot \nabla \psi=\left\langle\rho_{\eta}, \mathbf{v}_{\eta} \cdot \nabla \psi\right\rangle_{L^{2}\left(I ; H^{1}(\Omega)^{\prime}\right) \cap L^{2}\left(I ; H^{1}(\Omega)\right)} .
$$

Note that $\left(\rho_{\eta}\right)_{\eta \rightarrow 0}$ is uniformly bounded in the space $L^{2}\left(\Omega_{T}\right) \cap H^{1}\left(I ; W^{1, p}(\Omega)^{\prime}\right)$ for arbitrary $p>2$ if $d=2$, and for $p=3$ if $d=3$. Using $L^{2}(\Omega) \hookrightarrow \hookrightarrow H^{1}(\Omega)^{\prime} \hookrightarrow W^{1, p}(\Omega)^{\prime}$ and the Aubin-Lions lemma, we may pass to the limit $\eta \rightarrow 0$ to obtain for a subsequence,

$$
\lim _{\eta \rightarrow 0}\left\langle\rho_{\eta}, \mathbf{v}_{\eta} \cdot \nabla \psi\right\rangle_{L^{2}\left(I ; H^{1}(\Omega)^{\prime}\right) \cap L^{2}\left(I ; H^{1}(\Omega)\right)}=\langle\rho, \mathbf{v} \cdot \nabla \psi\rangle_{L^{2}\left(I ; H^{1}(\Omega)^{\prime}\right) \cap L^{2}\left(I ; H^{1}(\Omega)\right)}=\int_{\Omega_{T}} \rho \mathbf{v} \cdot \nabla \psi,
$$

which gives the result.

\section{Numerical experiments}

In this section, we present first numerical experiments in two space dimensions. For ease of implementation, we confine ourselves to system (4.1), i.e. we assume the effect of acceleration terms in the hydrodynamic equations to be small. In the present paper, we will only give a rather coarse description of the method; further details will be presented elsewhere.

\subsection{Discretization of the system}

For the convection-diffusion equations $4.1 \mathrm{~b}$ and $4.1 \mathrm{c}$, we use a finite-volume scheme. For the Stokes system, a mixed approximation based on stable Taylor-Hood elements has been implemented. The electrostatic potential $V$ is computed using standard FE-methods (see [13] and [9]).

The rectangular domains $\Omega$ and $\Omega^{*}$ are discretized by a simplicial triangulation with rectangular elements. We allow for heuristic adaptivity in space based on the magnitude of $|\nabla \phi|$ and $|\nabla \rho|$ as refinement criteria. In particular, this guarantees a refined spatial discretization in the diffuse interface region. In our experiments, the minimum grid size considered is given by $h \approx 5.5 \cdot 10^{-2}$, which would correspond in the case of a uniform triangulation to 33000 grid points.

For the convection-diffusion equations, we discretize diffusive terms implicitly and convective terms explicitly. For the latter, we combine Engquist-Osher methods with linear reconstruction and min-mod limiters to achieve higher order precision (cf. [20]). 
We decouple the non-stationary equations of (4.1) and use a semi-implicit splitting scheme. In the $k$-th time step, it reads as follows

- Find $\phi^{k+1 / 2}$ which solves $\frac{\phi^{k+1 / 2}-\phi^{k}}{\Delta t}=\nabla \cdot\left(M\left(\phi^{k+1 / 2}\right) \nabla \mu^{k+1 / 2}\right)$, where $\mu^{k+1 / 2}=-\delta \Delta \phi^{k+1 / 2}+$ $\frac{1}{\delta} W^{\prime}\left(\phi^{k+1 / 2}\right)-1 / 2 \varepsilon^{\prime}\left(\phi^{k}\right)\left|\nabla V^{k}\right|^{2}$.

- Find $V^{k+1}$ which solves $-\nabla\left(\bar{\varepsilon}\left[\phi^{k+1 / 2}\right] \nabla V^{k+1}\right)=\rho^{k}$.

- Find $\rho^{k+1 / 2}$ which solves $\frac{\rho^{k+1 / 2}-\rho^{k}}{\Delta t}=\nabla \cdot\left(K\left(\phi^{k+1 / 2}\right) \nabla\left(V^{k+1}+\lambda \rho^{k+1 / 2}\right)\right)$.

- Find $\mathbf{v}^{k+1}$ and $p^{k+1}$ which solve $-\nabla \cdot\left(\eta\left(\phi^{k+1 / 2}\right) \mathbf{T}\left(\mathbf{v}^{k+1}\right)\right)+\nabla p^{k+1}=\mu^{k+1 / 2} \nabla \phi^{k+1 / 2}-$ $\rho^{k+1 / 2} \nabla V^{k+1}$ and $\nabla \cdot \mathbf{v}^{k+1}=0$.

- Compute $\phi^{k+1}$ and $\rho^{k+1}$ via $\frac{\phi^{k+1}-\phi^{k+1 / 2}}{\Delta t}=-\mathbf{v}^{k+1} \cdot \nabla \phi^{k+1 / 2}$ and $\frac{\rho^{k+1}-\rho^{k+1 / 2}}{\Delta t}=-\mathbf{v}^{k+1} \cdot \nabla \rho^{k+1 / 2}$, respectively.

The arising non-linear system of equations is solved using Newton's method with Armijo rule. For the fourth order problem, a standard BiCGstab solver has been implemented (see [33]). The discrete Stokes problem gives rise to an indefinite Galerkin matrix; we use a preconditioned MinRES solver to compute the velocity field $\mathbf{v}$ (cf. [13]).

\subsection{Experiments}

Charged droplet on a surface. For our first experiments we consider a setting similar to Figure|A detailed sketch is given in Figure 4 . We embed the domain $\Omega$ into a larger domain $\Omega^{*}$. This is for two purposes. First, we need $\Omega^{*}$ to take the insulating and dielectric layer into account. Secondly, it enables us to solve the potential equation on a larger domain - this way reducing the bias caused by approximate boundary conditions for the electric field. Physically, the set $\Omega^{*} \backslash \Omega$ can be thought of as a solid, insulating box surrounding $\Omega$.

For the dielectric parameters in $\Omega^{*} \backslash \Omega$, we assume

$$
\varepsilon_{*}(x)= \begin{cases}100 & \text { if } x \in \Omega_{1}^{*}, \\ 1 & \text { if } x \in \Omega_{2}^{*} .\end{cases}
$$

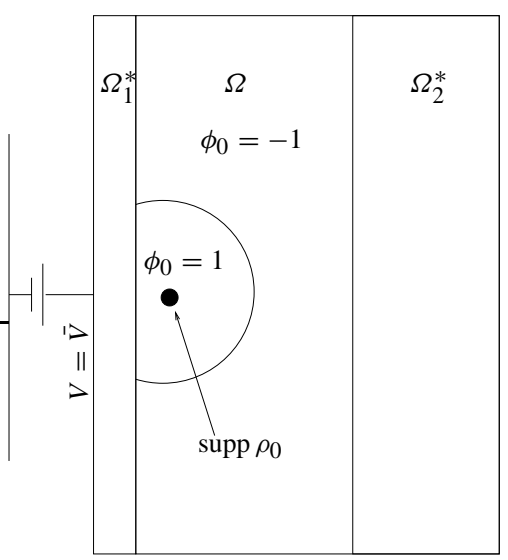

FIG. 4. Setting for the first experiment (charged droplet on a surface). The thickness of the dielectric layer $\Omega_{1}^{*}$ is $d=0.625$. Dimensions are $\Omega=[0,5] \times[-5,5], \Omega^{*}=\Omega_{1}^{*} \cup \Omega \cup \Omega_{2}^{*}$. 
As initial data, we choose

$$
\phi_{0}(x)=-\tanh \left(\frac{\sqrt{2}}{\delta}\left(|x|^{2}-1\right)\right) \text { and } \rho_{0}(x)=\rho_{0} \cdot \chi_{\Gamma_{0}}(x) .
$$

Here, $\Gamma_{0}$ stands for the initial support of charges and will be specified below.

For the electric conductivity $K(\phi)$ we take

$$
K(\phi)= \begin{cases}K_{-} & \text {if } \phi<0, \\ \frac{1}{2}\left(K_{-}+K_{+}+\left(K_{+}-K_{-}\right) \sin \left(\left(\phi-\frac{1}{2}\right) \pi\right)\right) & \text { if } 0 \leqslant \phi \leqslant 1, \\ K_{+} & \text {if } \phi>1,\end{cases}
$$

in accordance with $(\mathrm{H} 1)$ and $(\mathrm{H} 4)$, with values $\left(K_{-}, K_{+}\right)=\left(0,10^{3}\right)$.

The viscosity $\eta(\phi)$ is taken to be constant $(\eta \equiv 0.01)$, as is the mobility $(M \equiv 0.01)$ and the dielectric parameter in $\Omega(\varepsilon \equiv 1)$. We set $\alpha=0, \delta=0.1, \lambda=0.05$ and $\gamma_{0}=50$.

Here we present the results of three experiments with $\Delta \gamma_{f s}=\gamma_{f s}^{(1)}-\gamma_{f s}^{(2)} \in\left\{-\gamma_{0}, 0, \gamma_{0}\right\}$. This corresponds to equilibrium contact angles $\Theta(0)$ smaller than, equal to or larger than $\pi / 2$, respectively.

In absence of charges (left plot in Figures 5, 7 and 9), we observe a very quick evolution from the initial data given by half-circles to stationary states. The contact angle can be measured numerically - it turns out to be in accordance with Young's formula

$$
\gamma_{0} \cos \Theta(0)=\frac{3 \sqrt{2}}{8} \Delta \gamma_{f s} .
$$

If the initial charge is positive, the droplet starts to spread. In Figures 5, 7,and 9 , one can clearly see that the droplet flattens very quickly before it becomes nearly stationary. We observe a significant difference between contact angles on a macroscopic and a microscopic scale. In particular, the microscopic contact angle seems not to decrease during the evolution. Moreover, visual inspection indicates that for stationary states the Young angle persists. In fact, in view of boundary condition 2.19 this is no surprise. Note that 2.19) reduces in a quasi-steady state approximately to the identity $\gamma_{0} \delta \frac{\partial \phi}{\partial \mathbf{n}}+\gamma_{f s}^{\prime}(\phi)=0$. This is exactly the diffuse interface version of Young's formula for the equilibrium contact angle (cf. [31]). Hence, with regard to equilibrium configurations, our numerical results are in accordance with the observation by Mugele et al. (cf. [7] and [26]) mentioned in the introduction that the contact angle change predicted by Lippmann is merely a macroscopic effect.

During long-time computations, it might happen after the phase-field has become nearly stationary that charges move across the interface into the non-conductive phase. This effect seems to be due to the diffusivity of the interface-it strongly depends on the parameters chosen.

Charged droplet in a potential. The second class of experiments investigates the effect charges exert on an elliptic droplet between grounded electrodes. We take $\Omega=\Omega^{*}=[-5,5]^{2}$ and initial data

$$
\phi_{0}(x)=-\tanh \left(\frac{\sqrt{2}}{\delta}\left(\frac{x_{1}^{2}}{a^{2}}+\frac{x_{2}^{2}}{b^{2}}-1\right)\right)
$$

with $x=\left(x_{1}, x_{2}\right) \in \mathbb{R}^{2}$, where $a=3 / 2$ and $b=2 / 3$ are the major and minor semi-axes of an ellipse. The initial charge is given by

$$
\rho_{0}(x)=\rho_{0} \cdot\left|\Gamma_{0}\right|^{-1} \cdot \chi_{\Gamma_{0}}(x) \quad \text { where } \quad \Gamma_{0}=\{x \in \Omega:|x| \leqslant 1 / 4\},
$$

with $\rho_{0} \in\left\{2^{-1 / 2} Q, 2^{1 / 2} Q\right\}$ and $Q=2^{7 / 4} \pi \sqrt{\gamma_{0}}$. 

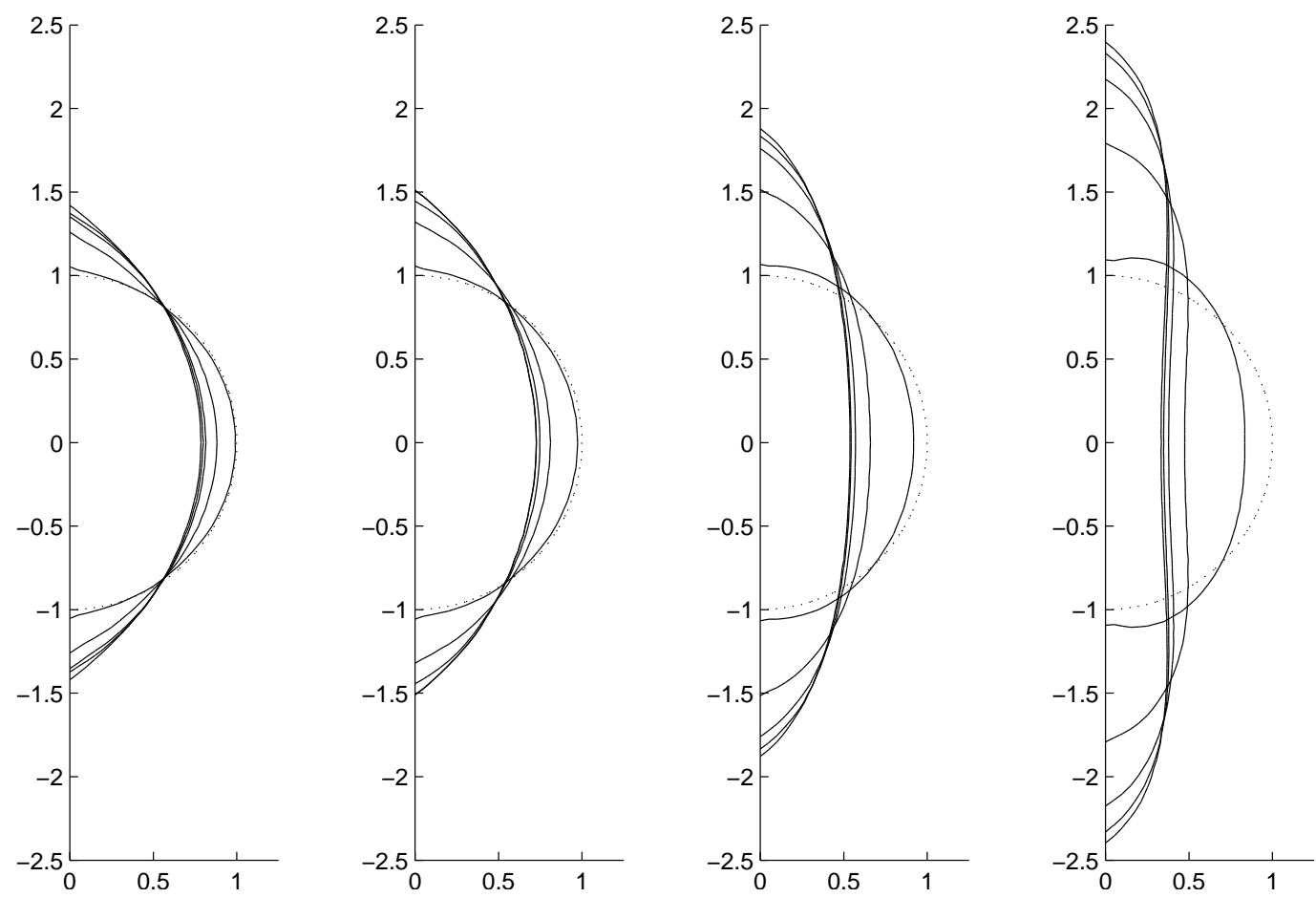

FIG. 5. Charged droplet on a partially wettable surface $\left(\Delta \gamma_{f s}=-\gamma_{0}, \Theta(0) \approx \frac{1}{3} \pi\right)$. The plots show the zero-level of $\phi$ at times $t=0$ (dashed), 0.001, 0.0025, 0.005, 0.0075, 0.01. Each plot shows a different choice of $\rho_{0}=0,200,400,600$ (from left to right).
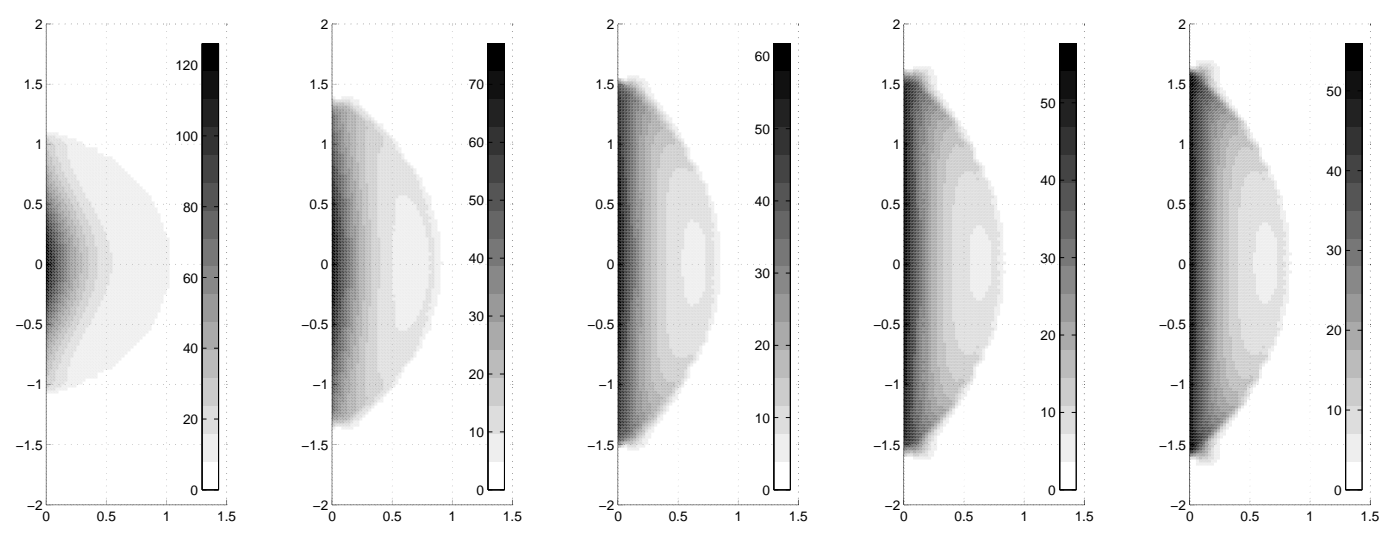

FIG. 6. Charged droplet on a partially wettable surface $\left(\Delta \gamma_{f s}=-\gamma_{0}, \Theta(0) \approx \frac{1}{3} \pi\right)$. The plots show the charge density $\rho$ at times $t=0.001,0.0025,0.005,0.0075,0.01$ (from left to right). $\rho_{0}=200$. 

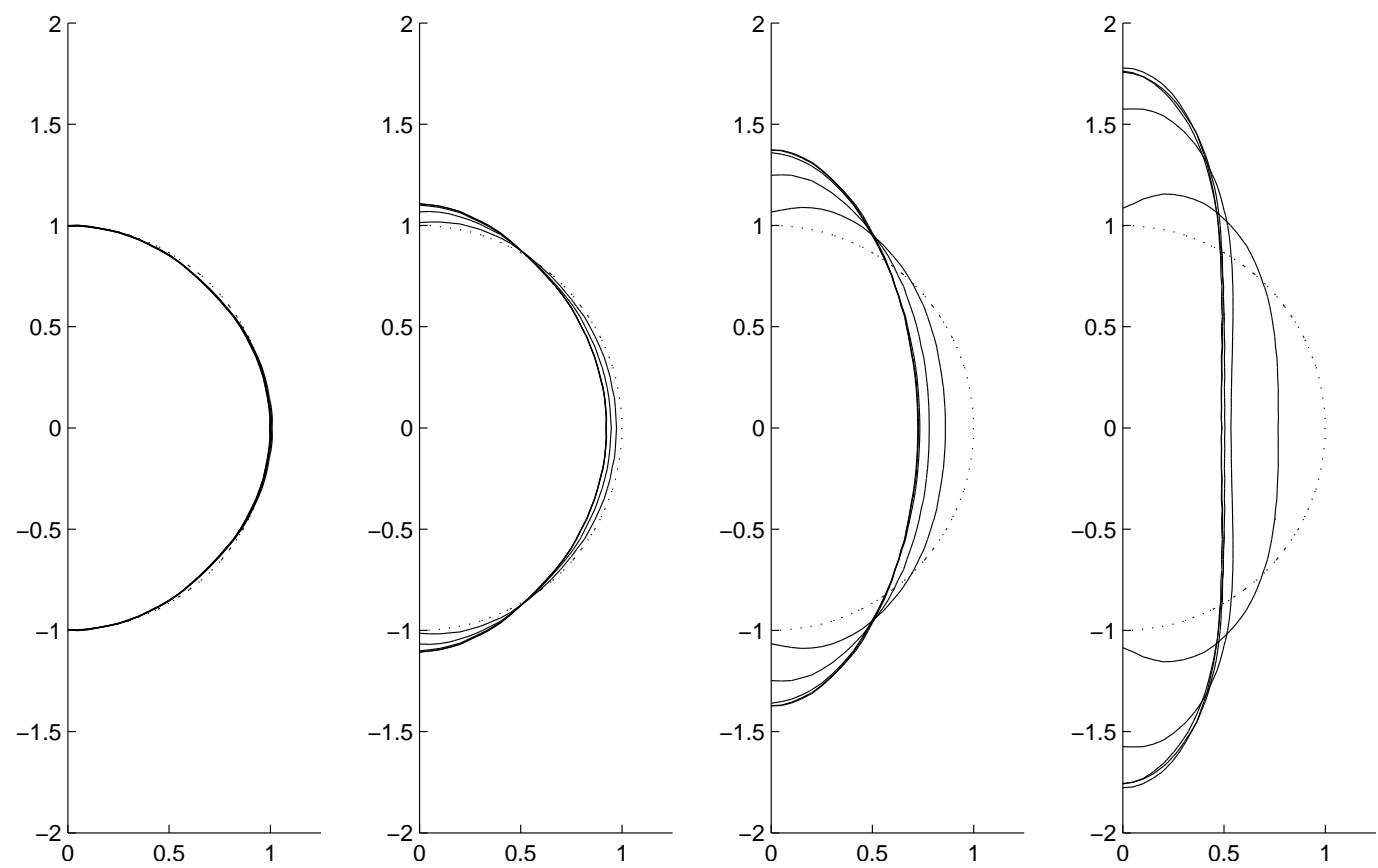

FIG. 7. Charged droplet on a surface $\left(\Delta \gamma_{f s}=0, \Theta(0)=\frac{\pi}{2}\right)$. The plots show the zero-level of $\phi$ at times $t=0$ (dashed), $0.001,0.0025,0.005,0.0075,0.01$. Each plot shows a different choice of $\rho_{0}=0,200,400,600$ (from left to right). Note also the temporary increase in the microscopic contact angle.

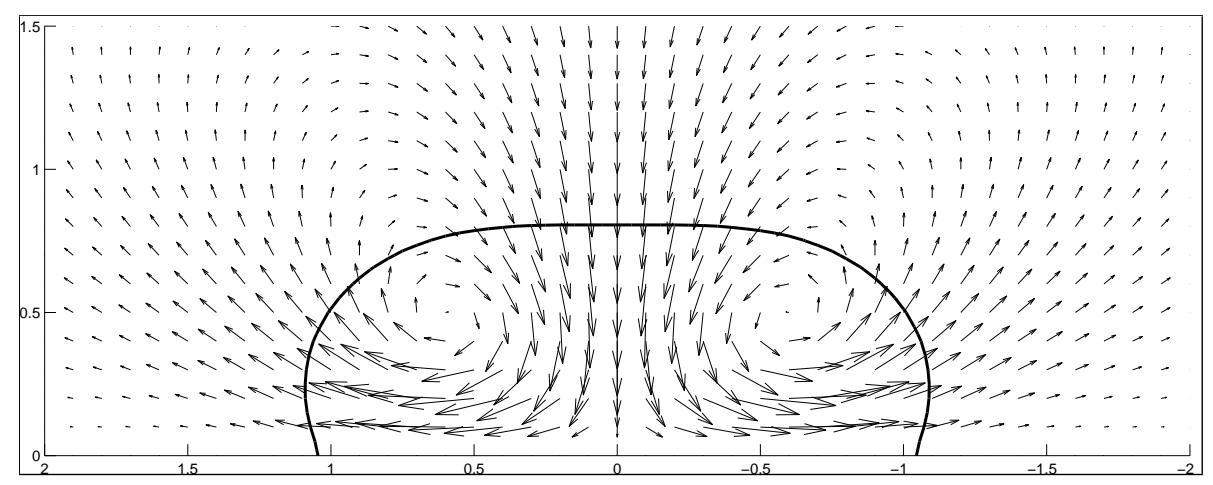

FIG. 8. Snapshot of the velocity field $\mathbf{v}$, corresponding to the sequence on the right of Figure 7

On the boundary of $\Omega^{*}$, we prescribe homogeneous Dirichlet boundary conditions for the potential $V$, and we take $\Delta \gamma_{f s}=0$. The other parameters are the same as in the first experiment.

We observe different evolution behavior depending on the amount of charge $\rho_{0}$ injected in the droplet. Having $\rho_{0}=2^{-1 / 2} Q$, the elliptic droplet evolves to a circular shape as seen in the left plot of Figure 10 Choosing a larger $\rho_{0}$ may cause the droplet to stay elliptic (right plot). A more detailed investigation of this probably critical value $Q$ will be presented elsewhere. 

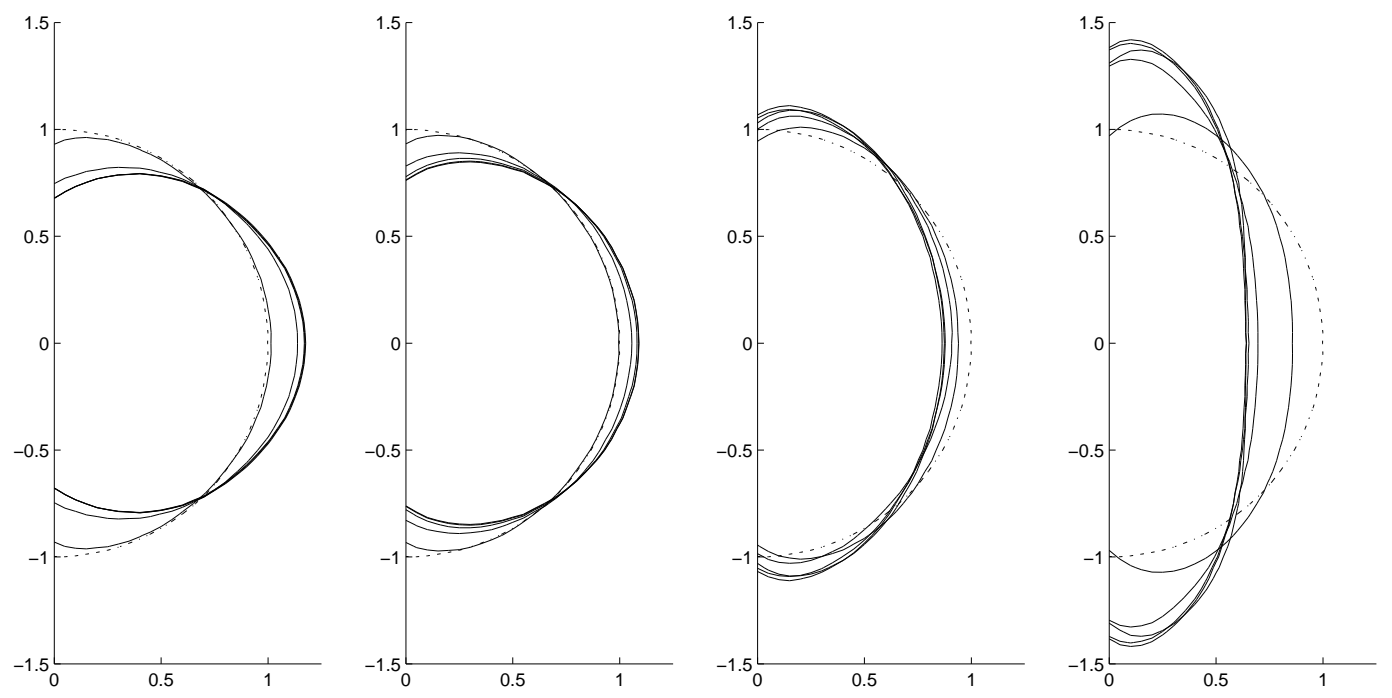

FIG. 9. Charged droplet on a hydrophobic surface $\left(\Delta \gamma_{f s}=\gamma_{0}, \Theta(0) \approx \frac{2}{3} \pi\right)$. The plots show the zero-level of $\phi$ at times $t=0$ (dashed), 0.001, 0.0025, 0.005, 0.0075, 0.01. Each plot shows a different choice of $\rho_{0}=0,200,400,600$ (from left to right).
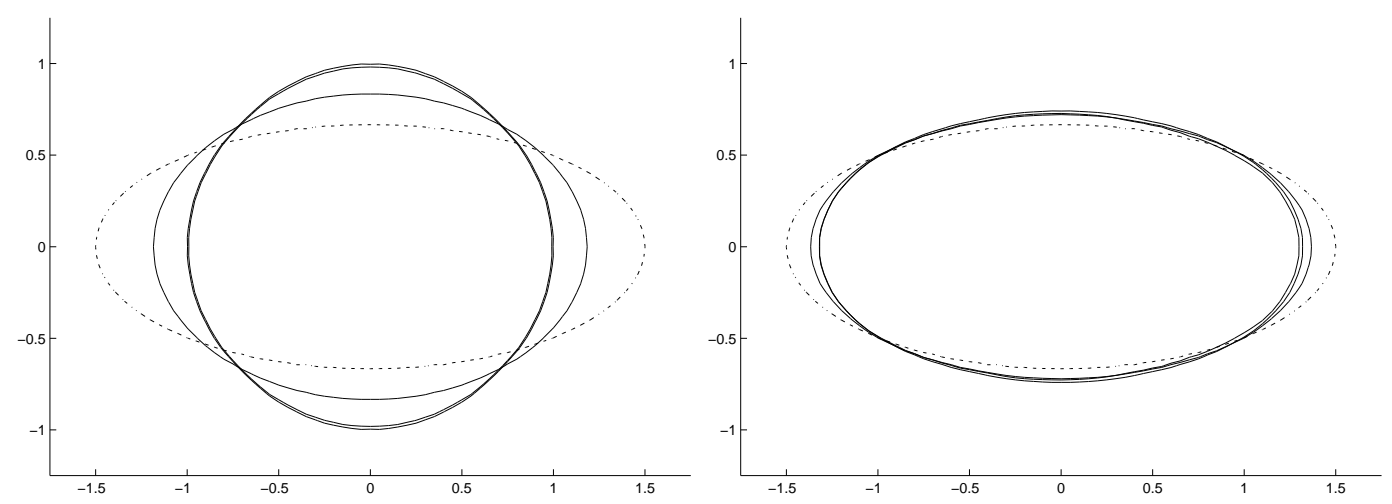

FIG. 10. Charged droplet in a potential. The plot shows the zero-level of $\phi$ at times $t=0$ (dashed), 0.0025, 0.005, 0.05. Each plot shows a different choice of $\rho_{0}=2^{-1 / 2} Q$ (left) and $\rho_{0}=2^{1 / 2} Q$ (right). The left plot (smaller $\rho_{0}$ ) shows the evolution to a circular shape, the right plot shows the evolution to an elliptic shape.

\section{Acknowledgements}

The authors gratefully acknowledge support by Deutscher Akademischer Austauschdienst and Ministerio de Educación y Ciencia through the program Acciones Integradas Hispano Alemanas, project D/06/12788. Parts of the paper have been written while C. Eck, G. Grün and F. Klingbeil visited Universidad Autónoma de Madrid and while M. Fontelos visited Friedrich-AlexanderUniversität Erlangen-Nürnberg. The warm hospitality of the host institutions is gratefully acknowledged by the authors. 


\section{REFERENCES}

1. ABELS, H. Existence of weak solutions for a diffuse interface model for viscous, incompressible fluids with general densities. Preprint, Max-Planck-Institute for Mathematics in the Sciences (2008).

2. Allen, S. M., \& CAHN, J. W. A macroscopic theory for antiphase boundary motion and its application to antiphase domain coarsening. Acta Metal. 27 (1979), 1085-1095.

3. Badalassi, V. E., Ceniceros, H. D., \& BanerJee, S. Computation of multiphase systems with phase field models. J. Comput. Phys. 190 (2003), 371-397. Zbl 1076.76517 MR 2013023

4. Bensoussan, A., \& Frehse, J. Regularity Results for Nonlinear Elliptic Systems and Applications. Springer, Berlin (2002).

5. Berge, B., \& Peseux, J. Variable focal lens controlled by an external voltage: An application of electrowetting. Eur. Phys. J. E: Soft Matter Biol. Phys. 3 (2000), 159-163.

6. Blake, T. D., Clarke, A., \& Stattersfield, E. H. An investigation of electrostatic assist in dynamic wetting. Langmuir 16 (2000), 2928-2935.

7. Bührle, J., Herminghaus, S., \& Mugele, F. Interface profiles near three-phase contact lines in electric fields. Phys. Rev. Lett. 91 (2003), 86101, 4 pp.

8. CAhn, J. W., \& Hilliard, J. E. Free energy of a nonuniform system, I. Interfacial free energy. J. Chem. Phys. 28 (1958), 258-267.

9. Ciarlet, P. G. The Finite Element Method for Elliptic Problems. North-Holland, Amsterdam (1978). Zbl 0999.65129 MR 1930132

10. Cooney, C. G., Chen, C. Y., Emerling, M., Nadim, A., \& Sterling, J. D. Electrowetting droplet microfluidics on a single planar surface, Microfluidics and Nanofluidics 2 (2006), 435-446.

11. Dacorogna, B. Weak Continuity and Weak Lower Semicontinuity of Nonlinear Functionals. Lecture Notes in Math. 922, Springer (1982). Zbl 0484.46041 MR 0658130

12. DiBenedetto, E. Degenerate Parabolic Equations. Springer (1993). Zbl 0794.35090 MR 1230384

13. Elman, H., Silvester, D., \& Wathen, A. Finite Elements and Fast Iterative Solvers with Applications in Incompressible Fluid Dynamics. Oxford Univ. Press, New York (2005) Zbl 1083.76001 MR 2155549

14. Feng, X. Fully discrete finite element approximations of the Navier-Stokes-Cahn-Hilliard diffuse interface model for two-phase fluid flows. SIAM J. Numer. Anal. 44 (2006), 1049-1072. Zbl pre05167765 MR 2231855

15. Feng, X., He, Y., \& LiU, C. Analysis of finite element approximations of a phase field model for two-phase fluids. Math. Comput. 76 (2007), 539-571. Zbl 1111.76028 MR 2291827

16. Gilbarg, D., \& Trudinger, N. S. Elliptic Partial Differential Equations of Second Order. Springer, Berlin (1983). Zbl 0562.35001 MR 0737190

17. Girault, V., \& Raviart, P. A. Finite Element Methods for Navier-Stokes Equations. Springer (1986). Zbl 0585.65077 MR 0851383

18. De Groot, S. R., \& MAZUR, P. Non-equilibrium Thermodynamics. North-Holland (1962). MR 0140332

19. JACKSON, J. D. Classical Electrodynamics. 3rd ed., Wiley (1999). Zbl 0920.00012

20. KRÖNER, D. Numerical Schemes for Conservation Laws. Wiley and Teubner (1997). Zbl 0872.76001 MR 1437144

21. Kuiper, S., \& HendriKs, B. H. W. Variable-focus liquid lens for miniature cameras. Appl. Phys. Lett. 85 (2004), 1128-1130.

22. Landau, L. D., Lifshitz, E. M., \& Pitaevskit, L. P. Electrodynamics of Continuous Media. 2nd ed., Academic Press (1984).

23. Lippmann, G. Ann. Chim. Phys. 5 (1875), 494. 
24. Lowengrub, J. S., \& Truskinovsky, L. Quasi-incompressible Cahn-Hilliard fluids and topological transitions. Proc. Roy. Soc. London A 454 (1998), 2617-2654. Zbl $0927.76007 \mid$ MR 1650795

25. Lu, H. W., Glasner, K., Bertozzi, A. L., \& Kim, C. J. A diffuse interface model for electrowetting drops in a Hele-Shaw cell. J. Fluid Mech. 590 (2007), 411-435. Z Zbl 1141.76482

26. Mugele, F., \& BüHrle, J. Equilibrium drop surface profiles in electric fields. J. Phys. Condens. Matter 19 (2007), 375112, 20 pp.; doi: 10.1088/0953-8984/19/37/375112.

27. Mugele, F., \& Herminghaus, S. Electrostatic stabilization of fluid microstructures. Appl. Phys. Lett. 81 (2002), 2303.

28. Navier, C. L. M. H. Mémoire sur les lois du movement des fluides. Mem. Acad. R. Sci. Inst. France 6 (1823), 389-440.

29. Onsager, L. Reciprocal relations in irreversible processes I. Phys. Rev. 37 (1931), 405-426. Zbl 0001.09501

30. Prins, M. W. J., Welters, W. J. J., \& Weekamp, J. W. Fluid control in multichannel structures by electrocapillary pressure. Science 291 (2001), 277-280.

31. QIAN, T., WANG, X. P., \& ShENG, P. A variational approach to moving contact line hydrodynamics. J. Fluid Mech. 564 (2006), 333-360. Zbl pre05068409 MR 2261865

32. RACKe, R., \& Zheng, S. The Cahn-Hilliard equation with dynamic boundary conditions. Adv. Differential Equations 8 (2003), 83-110. Zbl 1035.35050 MR 1946559

33. SAAD, Y. Iterative Methods for Sparse Linear Systems. 2nd ed., SIAM (2003). Zbl 1031.65046 MR 1990645

34. SAville, D. A. Electrohydrodynamics: The Taylor-Melcher leaky dielectric model. Ann. Rev. Fluid Mech. 29 (1997), 27-64. MR 1435033

35. Struwe, M. Variational Methods. Applications to Nonlinear Partial Differential Equations and Hamiltonian Systems. Springer, Berlin (1996). Zbl 0864.49001 MR 1411681

36. Vallet, M., VAllade, M., \& Berge, B. Limiting phenomena for the spreading of water on polymer films by electrowetting. Eur. Phys. J. B 11 (1999), 583-591.

37. Walker, S. W., \& Shapiro, B. Modeling the fluid dynamics of electrowetting on dielectric(EWOD). J. Microelectromech. Systems 15 (2006), 986-1000.

38. Young, T. An essay on the cohesion of fluids. Philos. Trans. Roy. Soc. London 95 (1805), 65-87. 